\title{
Boundary values of harmonic gradients and differentiability of Zygmund and Weierstrass functions
}

\author{
Juan J. Donaire, José G. Llorente and Artur Nicolau
}

\begin{abstract}
We study differentiability properties of Zygmund functions and series of Weierstrass type in higher dimensions. While such functions may be nowhere differentiable, we show that, under appropriate assumptions, the set of points where the incremental quotients are bounded has maximal Hausdorff dimension.
\end{abstract}

\section{Introduction and main results}

It was a widespread opinion among most of the mathematicians of the nineteenth century that a continuous function should be differentiable on a substantial set of points. For that reason, the first constructions of continuous nowhere differentiable functions on the real line - which go back to the end of the nineteenth century were not accepted without reservations. However, the existence of such pathological functions was a crucial breakthrough not only in the foundation of modern function theory but also in the future development of probability and physics. The first example of a continuous nowhere differentiable function is probably due to B. Bolzano (1830), who used a geometrical construction. However by the time Bolzano's example was published (1930) Weierstrass had already presented his construction in the Royal Academy of Berlin (1872, published in 1875). Some years earlier, Cellérier (1860, published in 1890) gave the first example by using a trigonometric series

$$
C(x)=\sum_{n=1}^{\infty} a^{-n} \sin \left(a^{n} x\right),
$$

Mathematics Subject Classification (2010): Primary 31B05, 31B25; Secondary 26B05.

Keywords: Zygmund class, Weierstrass functions, Hausdorff dimension. 
where $a>0$ is a sufficiently large number. Given $b>1$ and $0<\alpha \leq 1$, Weierstrass proved that the continuous function

$$
f_{b, \alpha}(x)=\sum_{n=0}^{\infty} b^{-n \alpha} \cos \left(2 \pi b^{n} x\right)
$$

is nowhere differentiable provided that $0<\alpha<1-\log (1+3 \pi / 2) / \log b$ and $b \geq 7$ is an odd integer. In 1916, Hardy ([9]) proved that the last restriction in Weierstrass's result was superfluous in the sense that $f_{b, \alpha}$ is nowhere differentiable as soon as $b>1$ and $0<\alpha \leq 1$. This is best possible and the extreme case $\alpha=1$ is the most delicate one.

During the twentieth century, a number of different geometric and analytic constructions of continuous nowhere differentiable functions have been obtained. See [18] for a historical survey of the subject.

For $0<\alpha \leq 1$, denote by $\operatorname{Lip}_{\alpha}\left(\mathbb{R}^{d}\right)$ the Hölder class of bounded functions $f: \mathbb{R}^{d} \rightarrow \mathbb{R}$ for which there exists a constant $C=C(f)>0$ such that $|f(x)-f(y)|<$ $C|x-y|^{\alpha}$ for any $x, y \in \mathbb{R}^{d}$. A standard trick in the theory of lacunary series gives that $f_{b, \alpha} \in \operatorname{Lip}_{\alpha}(\mathbb{R})$ if $0<\alpha<1$. On the other hand, a classical theorem of Rademacher (1919) says that any function in the Lipschitz class $\operatorname{Lip}_{1}\left(\mathbb{R}^{d}\right)$ is differentiable at almost every point. This implies in particular that $f_{b, 1}$ cannot be locally Lipschitz on any interval. However, $f_{b, 1}$ belongs to the so called Zygmund class. A bounded continuous function $f: \mathbb{R}^{d} \rightarrow \mathbb{R}$ is in the Zygmund class $\Lambda_{*}\left(\mathbb{R}^{d}\right)$ if

$$
\sup _{x, h} \frac{|f(x+h)+f(x-h)-2 f(x)|}{|h|}=\|f\|_{*}<\infty,
$$

where the supremum is taken over all $x \in \mathbb{R}^{d}$ and all $h \in \mathbb{R}^{d} \backslash\{0\}$. The Zygmund class is intermediate between the Hölder classes in the sense that $\operatorname{Lip}_{1}\left(\mathbb{R}^{d}\right) \subset$ $\Lambda_{*}\left(\mathbb{R}^{d}\right) \subset \operatorname{Lip}_{\alpha}\left(\mathbb{R}^{d}\right)$, for any $0<\alpha<1$. We also introduce the small Zygmund class $\lambda_{*}\left(\mathbb{R}^{d}\right)$ consisting of all bounded continuous functions $f: \mathbb{R}^{d} \rightarrow \mathbb{R}$ such that

$$
\sup _{x} \frac{|f(x+h)+f(x-h)-2 f(x)|}{|h|} \rightarrow 0 \quad \text { as }|h| \rightarrow 0 .
$$

Apart from its relation to Weierstrass functions, the Zygmund class is a convenient substitute of the Lipschitz class in some problems in harmonic analysis. See [1], [12], [13], [15], and [17] for connections between Zygmund classes, probability and other areas of analysis.

The main purpose of this paper is to discuss the behavior of the incremental quotients of a certain natural class of functions in the Zygmund class. We will need some notation. Given a function $f: \mathbb{R}^{d} \rightarrow \mathbb{R}$, define the sets

$$
\begin{aligned}
\mathcal{D}(f) & =\left\{x \in \mathbb{R}^{d}: \limsup _{|h| \rightarrow 0} \frac{|f(x+h)-f(x)|}{|h|}<\infty\right\}, \\
\mathcal{D}_{0}(f) & =\left\{x \in \mathbb{R}^{d}: f \text { is differentiable at } x\right\} .
\end{aligned}
$$


In [19], it was pointed out that if $f \in \Lambda_{*}(\mathbb{R})$ (resp. $\left.f \in \lambda_{*}(\mathbb{R})\right)$ then $\mathcal{D}(f)$ (resp. $\left.\mathcal{D}_{0}(f)\right)$ must be dense on any interval. On the other hand, from the classical theory of lacunary trigonometric series (see [20]) we have $m_{1}\left(\mathcal{D}\left(f_{b, 1}\right)\right)=0$ where, hereafter, $m_{d}$ denotes $d$-dimensional Lebesgue measure. From a metric point of view, the definitive answer in dimension $d=1$ was obtained in 1989 by Makarov (see [13] and [14]).

Theorem A (Makarov).

1) If $f \in \Lambda_{*}(\mathbb{R})$ then $\operatorname{Dim}(\mathcal{D}(f) \cap I)=1$ for any interval $I \subset \mathbb{R}$.

2) If $f \in \lambda_{*}(\mathbb{R})$ then $\operatorname{Dim}\left(\mathcal{D}_{0}(f) \cap I\right)=1$ for any interval $I \subset \mathbb{R}$.

Here and hereafter, Dim denotes Hausdorff dimension. Points in $\mathcal{D}(f)$ are sometimes called slow points of $f$ (see [11] for a version of Theorem A in the case of Brownian motion). The authors asked whether $\mathcal{D}(f)$ (respectively $\mathcal{D}_{0}(f)$ ) should also have maximal Hausdorff dimension if $f \in \Lambda_{*}\left(\mathbb{R}^{d}\right)$ (respectively $f \in \lambda_{*}\left(\mathbb{R}^{d}\right)$ ). In previous work ([7]) they showed that this is not the case: the right dimension is 1 and this is the best that can be said in general.

\section{Theorem B ([7]).}

1) If $f \in \Lambda_{*}\left(\mathbb{R}^{d}\right)$ then $\operatorname{Dim}(\mathcal{D}(f) \cap Q) \geq 1$ for any cube $Q \subset \mathbb{R}^{d}$.

2) If $f \in \lambda_{*}\left(\mathbb{R}^{d}\right)$ then $\operatorname{Dim}\left(\mathcal{D}_{0}(f) \cap Q\right) \geq 1$ for any cube $Q \subset \mathbb{R}^{d}$.

3) There is $f \in \lambda_{*}\left(\mathbb{R}^{d}\right)$ such that $\operatorname{Dim}(\mathcal{D}(f))=1$.

In this paper we will mainly focus on differentiability properties of Weierstrass type functions. Our method can be presented in two steps: i) give sufficient conditions on a function $f \in \Lambda_{*}\left(\mathbb{R}^{d}\right)$ implying that $\operatorname{Dim}(\mathcal{D}(f))=d$; ii) show that a certain class of Weierstrass type functions satisfies the previous sufficient conditions.

Regarding i), our method is based on a principle that has been known for a long time: there is a correspondence between the differentiability properties of a function $f: \mathbb{R}^{d} \rightarrow \mathbb{R}$ and the boundary behavior of $\nabla F: \mathbb{R}_{+}^{d+1} \rightarrow \mathbb{R}^{d+1}$, where $F: \mathbb{R}_{+}^{d+1} \rightarrow \mathbb{R}$ is the Poisson extension of $f$ to the upper half-space $\mathbb{R}_{+}^{d+1}$. See the results in Section 2. Let $F: \mathbb{R}_{+}^{d+1} \rightarrow \mathbb{R}$ be a harmonic function. We say that the gradient vector field $\nabla F: \mathbb{R}_{+}^{d+1} \rightarrow \mathbb{R}^{d+1}$ is Bloch (resp. little Bloch), written $\nabla F \in \mathcal{B}\left(\mathbb{R}_{+}^{d+1}\right)\left(\right.$ resp. $\left.\nabla F \in \mathcal{B}_{0}\left(\mathbb{R}_{+}^{d+1}\right)\right)$ if

$$
\sup _{(x, y)} y\|H F(x, y)\|<\infty,
$$

where $H F(x, y)=D(\nabla F(x, y))$ is the Hessian of $F$ at $(x, y)$ and the supremum is taken over all $(x, y) \in \mathbb{R}_{+}^{d+1}\left(\right.$ resp. $\sup _{x} y\|H F(x, y)\| \rightarrow 0$ as $\left.y \rightarrow 0\right)$. Geometrically, the Bloch condition says that the oscillation of $\nabla F$ in regions of $\mathbb{R}_{+}^{d+1}$ of a fixed hyperbolic diameter is uniformly bounded (see Proposition 2.1). 
For harmonic $F: \mathbb{R}_{+}^{d+1} \rightarrow \mathbb{R}$, define, analogously to (1.1) and (1.2):

$$
\begin{aligned}
\mathcal{D}(\nabla F) & =\left\{x \in \mathbb{R}^{d}: \limsup _{y \rightarrow 0}|\nabla F(x, y)|<\infty\right\}, \\
\mathcal{D}_{0}(\nabla F) & =\left\{x \in \mathbb{R}^{d}: \lim _{y \rightarrow 0} \nabla F(x, y) \text { exists }\right\} .
\end{aligned}
$$

Then Theorem A can be deduced from the following stronger result of Makarov (see Section 5 of Chapter II in [14]).

Theorem C. Let $F: \mathbb{R}_{+}^{2} \rightarrow \mathbb{R}$ be a harmonic function.

1) If $\nabla F \in \mathcal{B}\left(\mathbb{R}_{+}^{2}\right)$, then $\operatorname{Dim}(\mathcal{D}(\nabla F) \cap I)=1$ for any interval $I \subset \mathbb{R}$.

2) If $\nabla F \in \mathcal{B}_{0}\left(\mathbb{R}_{+}^{2}\right)$, then $\operatorname{Dim}\left(\mathcal{D}_{0}(\nabla F) \cap I\right)=1$ for any interval $I \subset \mathbb{R}$.

Corollary 2.4 says that $\mathcal{D}(f)=\mathcal{D}\left(\nabla_{x} F\right)$, where $\nabla_{x} F$ denotes the tangential component of $\nabla F$. Consequently Theorem $\mathrm{C}$ is stronger than Theorem A since the result affects the two derivatives and not only $\partial F / \partial x$. A decisive feature in the proof of Theorem $\mathrm{C}$ is that, since $d=1, \nabla F$ is an anti-analytic function so in particular $H F(x, y)$ is a conformal matrix for each $(x, y)$. That means that $\nabla F$ distorts in the same way in different directions, a fact which plays a role in the proof of Theorem C.

More generally, we say that a smooth mapping $G: \Omega \rightarrow \mathbb{R}^{n}, \Omega \subset \mathbb{R}^{n}$ is quasiregular if there exists a constant $0<K<\infty$ such that

$$
\max _{|e|=1}|D G(x)(e)| \leq K \min _{|e|=1}|D G(x)(e)|
$$

for any $x \in \Omega$. Quasiregularity implies that, infinitesimally, $G$ distorts about the same in the different directions. See [2], [16] for an account on the theory of quasiregular mappings, under much milder regularity assumptions. If $F$ is harmonic in $\mathbb{R}_{+}^{2}$ then $\nabla F$ is quasi-regular with constant 1 . On the other hand, in dimensions greater or equal than $3, K=1$ in (1.5) implies that $D G(x)$ is a conformal matrix in which case $G$ is in fact a linear conformal transformation by a classical result of Liouville (see [16]). In Section 4 we will use a weaker notion of quasi-regularity. Let $F: \mathbb{R}_{+}^{d+1} \rightarrow \mathbb{R}$ be a harmonic function. We say that $\nabla F$ is weakly quasiregular if there exist an integer $N \geq 2$ and $\gamma \geq 1$ such that $\nabla F$ satisfies $1 / N$-weak quasiregularity condition with constant $\gamma$,

$$
\int_{C_{1 / N}(Q)} \max _{|e|=1}|(H F) e(x, y)|^{2} d x d y \leq \gamma^{2} \min _{|e|=1} \int_{C_{1 / N}(Q)}|(H F) e(x, y)|^{2} d x d y
$$

for any cube $Q \subset \mathbb{R}^{d}$ of sidelength $l(Q)$, where $C_{1 / N}(Q)=Q \times[l(Q) / N, l(Q)] \subset$ $\mathbb{R}_{+}^{d+1}$ is the $1 / N$-Carleson box associated to the cube $Q \subset \mathbb{R}^{d}, H F(x, y)$ is the Hessian of $F$ at $(x, y)$ and the maximum and the minimum are taken over all unitary vectors $e \in \mathbb{R}^{d+1}$. Obviously, quasiregularity is stronger than weak quasiregularity, in the above sense. Our first main result says that, assuming weak quasi-regularity, Theorem $\mathrm{C}$ can be generalized to any dimension. 
Theorem 1. Let $F: \mathbb{R}_{+}^{d+1} \rightarrow \mathbb{R}$ be a harmonic function. Assume that $\nabla F$ is weakly quasiregular.

1) If $\nabla F \in \mathcal{B}\left(\mathbb{R}_{+}^{d+1}\right)$ then $\operatorname{Dim}(\mathcal{D}(\nabla F) \cap Q)=d$ for any cube $Q \subset \mathbb{R}^{d}$.

2) If $\nabla F \in \mathcal{B}_{0}\left(\mathbb{R}_{+}^{d+1}\right)$ then $\operatorname{Dim}\left(\mathcal{D}_{0}(\nabla F) \cap Q\right)=d$ for any cube $Q \subset \mathbb{R}^{d}$.

As explained above, we obtain the following consequence which should be compared with Theorem B.

Corollary 2. Let $f: \mathbb{R}^{d} \rightarrow \mathbb{R}$ be a bounded continuous function and let $F$ be its Poisson extension to $\mathbb{R}_{+}^{d+1}$. Assume that $\nabla F$ is weakly quasiregular.

1) If $f \in \Lambda_{*}\left(\mathbb{R}^{d}\right)$ then $\operatorname{Dim}(\mathcal{D}(f) \cap Q)=d$ for any cube $Q \subset \mathbb{R}^{d}$.

2) If $f \in \lambda_{*}\left(\mathbb{R}^{d}\right)$ then $\operatorname{Dim}\left(\mathcal{D}_{0}(f) \cap Q\right)=d$ for any cube $Q \subset \mathbb{R}^{d}$.

The rest of the results deal with specific examples of Zygmund functions given by Weierstrass series. We have adapted ideas from a recent paper of Y. Heurteaux ([10]) where he studies the nowhere differentiability of Weierstrass-type functions on the real line.

For $\varepsilon>0$, let $\mathcal{C}^{1, \varepsilon}\left(\mathbb{R}^{d}\right)$ be the class of bounded functions $f: \mathbb{R}^{d} \rightarrow \mathbb{R}$ for which there exists a constant $C=C(f)>0$ such that $|f(x+h)+f(x-h)-2 f(x)|<$ $C|h|^{1+\varepsilon}$ for any $x, h \in \mathbb{R}^{d}$. When $0<\varepsilon<1$, the class $\mathcal{C}^{1, \varepsilon}\left(\mathbb{R}^{d}\right)$ consists of the differentiable functions whose first partial derivatives belong to the Hölder class $\operatorname{Lip}_{\varepsilon}\left(\mathbb{R}^{d}\right)$. Also $\mathcal{C}^{2, \varepsilon}\left(\mathbb{R}^{d}\right)$ is the class of functions whose first partial derivatives are in $\mathcal{C}^{1, \varepsilon}\left(\mathbb{R}^{d}\right)$. Let $\phi: \mathbb{R}^{d} \rightarrow \mathbb{R}$ be a function of class $\mathcal{C}^{1, \varepsilon}\left(\mathbb{R}^{d}\right)$ which is 1 -periodic in each coordinate, that is,

$$
\phi\left(x_{1}, \ldots, x_{i}+1, \ldots, x_{d}\right)=\phi\left(x_{1}, \ldots, x_{d}\right)
$$

for any $x=\left(x_{1}, \ldots, x_{d}\right) \in \mathbb{R}^{d}$ and for each $i=1, \ldots, d$. For $b>1$ define the Weierstrass function associated to $b$ and $\phi$ by

$$
f_{b, \phi}(x)=\sum_{n=0}^{\infty} b^{-n} \phi\left(b^{n} x\right) .
$$

In dimension $d=1, \mathrm{Y}$. Heurteaux has proved in $[10]$ that either $f_{b, \phi} \in \mathcal{C}^{1, \varepsilon}(\mathbb{R})$ (and hence it is differentiable at every point) or $f_{b, \phi}$ is nowhere differentiable. This dichotonomy extends easily to dimension $d>1$. Heurteaux also gives the following sufficient condition.

Theorem D. Let $d=1$ and $b>1$ and let $\phi$ and $f_{b, \phi}$ be as above. Assume that either i) $\phi^{\prime}(0) \neq 0$ or $\left.\mathrm{ii}\right) \phi$ is nonconstant and has a global extremum at $t=0$. Then $f_{b, \phi}$ is nowhere differentiable.

Similarly, we will say that a differentiable function $\phi: \mathbb{R}^{d} \rightarrow \mathbb{R}$ satisfies condition $\mathcal{H}$ if, for each unitary vector $e \in \mathbb{R}^{d}$, either $D_{e} \phi(0) \neq 0$ or the one-variable function $t \rightarrow \phi(t e)$ is nonconstant and has a global extremum at $t=0$. Our main result is the following. 
Theorem 3. Let $\phi: \mathbb{R}^{d} \rightarrow \mathbb{R}$ be a function of class $\mathcal{C}^{2, \alpha}\left(\mathbb{R}^{d}\right)$ for some $0<\alpha<1$ which is 1-periodic in each coordinate. For $b>1$ let $f_{b, \phi}$ be the Weierstrass function associated to $b$ and $\phi$ as in (1.7). Assume in addition that $\phi$ satisfies condition $\mathcal{H}$. Then:

1) $f_{b, \phi} \in \Lambda_{*}\left(\mathbb{R}^{d}\right)$ and $f_{b, \phi}$ is nowhere differentiable.

2) For any unitary vector $e \in \mathbb{R}^{d}$ we have

$$
m_{d}\left\{x \in \mathbb{R}^{d}: \limsup _{t \rightarrow 0} \frac{\left|f_{b, \phi}(x+t e)-f_{b, \phi}(x)\right|}{|t|}<\infty\right\}=0 .
$$

In particular $m_{d}\left(\mathcal{D}\left(f_{b, \phi}\right)\right)=0$.

3) $\operatorname{Dim}\left(\mathcal{D}\left(f_{b, \phi}\right) \cap Q\right)=d$ for any cube $Q \subset \mathbb{R}^{d}$.

The most relevant result in Theorem 3 is part 3), which should be compared with Theorem B. The key point is to show that if $F$ is the harmonic extension of $f_{b, \phi}$ to $\mathbb{R}_{+}^{d+1}$ then condition $\mathcal{H}$ implies a certain uniform lower bound on $H F$ (Lemma 7.3 below), which is the substitute for the oscillation condition in Theorem 1.2 in [10]. From such uniform lower bound, it is easy to deduce that $\nabla F$ is weakly quasi-regular, which makes it possible to apply Theorem 1.

The paper is organized as follows. Section 2 contains some basic facts about Zygmund functions and their connections with harmonic extensions. Section 3 describes how to use stopping-time methods to construct Cantor-like boundary sets at which a gradient Bloch vector field is bounded. Section 4 shows how the weak quasi-regularity condition guarantees that the boundary sets in Section 3 have large Hausdorff dimension. In Section 5, Theorem 1, part 1) is proved and a sketch of the proof of part 2) is given. Section 6 contains some standard facts about regularity of Poisson extensions. Section 7 is devoted to functions of Weierstrass type in higher dimensions. Theorem 3 is proved in Section 8. Finally, Section 9 includes some remarks and questions.

\section{Some properties of Zygmund functions and their harmonic extensions}

If $f: \mathbb{R}^{d} \rightarrow \mathbb{R}$ and $f \in L^{\infty}\left(\mathbb{R}^{d}\right)$, recall that the harmonic extension of $f$ to the upper half-space $\mathbb{R}_{+}^{d+1}$ is given by

$$
F(x, y)=\int_{\mathbb{R}^{d}} P(x-z, y) f(z) d z \quad\left(x \in \mathbb{R}^{d}, y>0\right)
$$

where

$$
P(x, y)=c_{d} \frac{y}{\left(|x|^{2}+y^{2}\right)^{(d+1) / 2}}, \quad c_{d}=\frac{\Gamma((d+1) / 2)}{\pi^{(d+1) / 2}},
$$

is the Poisson kernel in the upper half-space. 
We say that a harmonic function $v$ in the upper half space $\mathbb{R}_{+}^{d+1}$ is a Bloch function, denoted $v \in \mathcal{B}\left(\mathbb{R}_{+}^{d+1}\right)$, if and only if

$$
\sup \left\{y|\nabla v(x, y)|:(x, y) \in \mathbb{R}_{+}^{d+1}\right\}=\|v\|_{\mathcal{B}}<\infty \text {. }
$$

If

$$
\sup \left\{y|\nabla v(x, y)|: x \in \mathbb{R}^{d}\right\} \rightarrow 0 \quad \text { as } y \rightarrow 0,
$$

then we say that $v$ belongs to the little Bloch class and write $v \in \mathcal{B}_{0}\left(\mathbb{R}_{+}^{d+1}\right)$. The following proposition is elementary.

Proposition 2.1. Let $v \in \mathcal{B}\left(\mathbb{R}_{+}^{d+1}\right)$. If $a, b \in \mathbb{R}^{d}$ and $s, t>0$ then

$$
|v(b, t)-v(a, s)| \leq\|v\|_{\mathcal{B}}\left(\frac{|b-a|}{\max \{t, s\}}+\left|\log \left(\frac{t}{s}\right)\right|\right) .
$$

Proof. Suppose that $0<s \leq t$. Then

$$
|v(b, t)-v(a, s)| \leq|v(b, t)-v(a, t)|+|v(a, t)-v(a, s)| .
$$

Use the Bloch condition on each term.

If $F$ is harmonic in $\mathbb{R}_{+}^{d+1}$ we say that $\nabla F \in \mathcal{B}\left(\mathbb{R}_{+}^{d+1}\right.$ ) (respectively $\nabla F \in$ $\left.\mathcal{B}_{0}\left(\mathbb{R}_{+}^{d+1}\right)\right)$ if all the partial derivatives $\partial F / \partial x_{i}, \partial F / \partial y, i=1, \ldots, d$ are Bloch (resp. little Bloch). Whenever $\nabla F \in \mathcal{B}\left(\mathbb{R}_{+}^{d+1}\right)$, we also write

$$
\|\nabla F\|_{\mathcal{B}}=\sup \left\{y\left|\frac{\partial^{2} F}{\partial x_{i} \partial x_{j}}(x, y)\right|:(x, y) \in \mathbb{R}_{+}^{d+1}, i, j=1, \ldots, d+1\right\},
$$

where, for simplicity, $x_{d+1}$ denotes the $y$-variable. The following proposition, whose proof can be found on p. 146 of [17], relates the Zygmund and Bloch classes.

Proposition 2.2. Let $f \in L^{\infty}\left(\mathbb{R}^{d}\right)$ and let $F$ be its Poisson extension to the upper half-space $\mathbb{R}_{+}^{d+1}$. Then $f \in \Lambda_{*}\left(\mathbb{R}^{d}\right)$ if and only if $\nabla F \in \mathcal{B}\left(\mathbb{R}_{+}^{d+1}\right)$. Moreover there exists a positive constant $C$ depending only on $d$ such that

$$
C^{-1}\|f\|_{*} \leq\|\nabla F\|_{\mathcal{B}} \leq C\|f\|_{*} .
$$

Furthermore, $f \in \lambda_{*}\left(\mathbb{R}^{d}\right)$ if and only if $\nabla F \in \mathcal{B}_{0}\left(\mathbb{R}_{+}^{d+1}\right)$.

The following two propositions relate the incremental quotients of Zygmund functions to the vertical behavior of the tangential components of the gradients of their Poisson extensions. Given a smooth function $F: \mathbb{R}_{+}^{d+1} \rightarrow \mathbb{R}$, the tangential component of its gradient is $\nabla_{x} F=\left(\partial F / \partial x_{1}, \ldots, \partial F / \partial x_{d}\right)$.

Proposition 2.3. Let $f \in \Lambda_{*}\left(\mathbb{R}^{d}\right)$ and let $F$ be its Poisson extension to $\mathbb{R}_{+}^{d+1}$. Then, for any $x, h \in \mathbb{R}^{d}, h \neq 0$, we have

$$
\frac{\left|f(x+h)-f(x)-h \cdot \nabla_{x} F(x,|h|)\right|}{|h|} \leq C\|f\|_{*} .
$$


Proof. We will use the following representation of $f$, which can be checked by differentiation: for any $y>0$,

$$
f(x)=F(x, y)-y \frac{\partial F}{\partial y}(x, y)+\int_{0}^{y} t \frac{\partial^{2} F}{\partial y^{2}}(x, t) d t .
$$

Choose $y=|h|$ so that $f(x+h)-f(x)-h \cdot \nabla_{x} F(x,|h|)=\Delta_{1}-\Delta_{2}+\Delta_{3}$, where

$$
\begin{aligned}
& \Delta_{1}=F(x+h,|h|)-F(x,|h|)-h \cdot \nabla_{x} F(x,|h|), \\
& \Delta_{2}=|h|\left[\frac{\partial F}{\partial y}(x+h,|h|)-\frac{\partial F}{\partial y}(x,|h|)\right], \\
& \Delta_{3}=\int_{0}^{|h|} t\left(\frac{\partial^{2} F}{\partial y^{2}}(x+h, t)-\frac{\partial^{2} F}{\partial y^{2}}(x, t)\right) d t .
\end{aligned}
$$

Clearly $\left|\Delta_{3}\right| \leq 2\|\nabla F\|_{\mathcal{B}}|h|$, and Proposition 2.1 gives that $\left|\Delta_{2}\right| \leq\|\nabla F\|_{\mathcal{B}}|h|$. On the other hand, also from Proposition 2.1,

$$
\left|\Delta_{1}\right|=\mid \int_{0}^{1} h \cdot\left(\nabla_{x} F(x+t h,|h|)-\nabla_{x} F(x,|h|) d t\left|\leq\|\nabla F\|_{\mathcal{B}}\right| h \mid,\right.
$$

and the result follows from Proposition 2.2.

The following result follows easily.

Corollary 2.4. Let $f \in \Lambda_{*}\left(\mathbb{R}^{d}\right)$. Then

$$
\limsup _{|h| \rightarrow 0} \frac{|f(x+h)-f(x)|}{|h|}<\infty \Longleftrightarrow \limsup _{y \rightarrow 0}\left|\nabla_{x} F(x, y)\right|<\infty .
$$

The analogues of Proposition 2.3 and Corollary 2.4 for the little Zygmund class read as follows and are proved in the same way.

Proposition 2.5. Let $f \in \lambda_{*}\left(\mathbb{R}^{d}\right)$ and let $F$ be its Poisson extension to $\mathbb{R}_{+}^{d+1}$. Then for any $x \in \mathbb{R}^{d}$, one has

$$
\lim _{h \rightarrow 0} \frac{f(x+h)-f(x)-h \cdot \nabla_{x} F(x,|h|)}{|h|}=0 .
$$

Corollary 2.6. Let $f$ and $F$ be as in Proposition 2.5. Then

$$
f \text { is differentiable at } x \Longleftrightarrow \lim _{y \rightarrow 0} \nabla_{x} F(x, y) \text { exists. }
$$

\section{On the boundedness of Bloch gradients at the boundary}

Let $F$ be harmonic in $\mathbb{R}_{+}^{d+1}$ such that $\nabla F$ is Bloch (for instance $F$ could be the Poisson extension of a Zygmund function in $\mathbb{R}^{d}$ ). We are interested in the size of the set

$$
\mathcal{D}(\nabla F)=\left\{x \in \mathbb{R}^{d}: \lim \sup _{y \rightarrow 0}|\nabla F(x, y)|<\infty\right\}
$$


Since this is largely a technical section, let us briefly explain its content. In order to determine conditions under which the set $\mathcal{D}(\nabla F)$ has Hausdorff dimension $d$, the strategy is to construct a Cantor-like subset of $\mathcal{D}(\nabla F)$ by using stopping-time constructions (Proposition 3.3) and to try to estimate its Hausdorff dimension from below. It turns out that if the stopping time satisfies a certain homogeneity covering condition (see equation (3.9) below) then we get good control on the Hausdorff dimension of such a Cantor-like set and we will be able to deduce (Corollary 3.8) that $\operatorname{Dim}(\mathcal{D}(\nabla F))=d$.

Proposition 3.1. Let $F$ be a harmonic function in $\mathbb{R}_{+}^{d+1}$ such that $\nabla F \in \mathcal{B}\left(\mathbb{R}_{+}^{d+1}\right)$. Then

$$
\mathcal{D}(\nabla F)=\left\{x \in \mathbb{R}^{d}: \lim _{y \rightarrow 0} \nabla F(x, y) \text { exists }\right\} \cup N,
$$

where $N$ has d-dimensional measure zero. In particular, if $F$ is the Poisson extension of a function $f \in \Lambda_{*}\left(\mathbb{R}^{d}\right)$ and

$$
\limsup _{h \rightarrow 0} \frac{|f(x+h)-f(x)|}{|h|}=\infty \quad \text { a.e. } x \in \mathbb{R}^{d},
$$

then $\mathcal{D}(\nabla F)$ has zero d-dimensional measure.

Proof. From Proposition 2.1, $\nabla F$ is nontangentially bounded at any point of $\mathcal{D}(\nabla F)$. From the local Fatou theorem for harmonic functions (see Theorem 3 in Chapter VII of [17]), it follows that for almost all points $x \in \mathcal{D}(\nabla F)$, the limit $\lim _{y \rightarrow 0} \nabla F(x, y)$ exists. The second implication follows from Corollary 2.4.

Since $\mathcal{D}(\nabla F)$ could have $d$-dimensional Lebesgue measure zero, we may ask what can be said about its Hausdorff dimension. The purpose of Sections 3 and 4 is to establish that, if $\nabla F$ satisfies a certain quasiregularity assumption, then $\mathcal{D}(\nabla F)$ has Hausdorff dimension $d$. In dimension $d=1$, this was proved by N. Makarov ([13], [14]). Observe that if $d=1$, the quasiregularity assumption is always fulfilled.

One can obtain satisfactory lower bounds of the Hausdorff dimension of sets of Cantor type, as the following lemma shows. It is a well known higher-dimensional version of a lemma of Hungerford (see [15], Theorem 10.5). Hereafter, $l(Q)$ stands for the sidelength of a cube $Q$.

Lemma 3.2. Let $\alpha, \beta>0$ with $\alpha<\beta^{1 / d}<1$. For $k=0,1,2 \ldots$, let $E_{k}$ be $a$ countable union of disjoint cubes $\left\{Q_{j}^{k}: j=1,2, \ldots\right\}$ in $\mathbb{R}^{d}$. Suppose that for any $k=0,1,2, \ldots$ the following two conditions hold:

1) Whenever $Q_{i}^{k+1} \cap Q_{j}^{k} \neq \emptyset$ then $Q_{i}^{k+1} \subset Q_{j}^{k}$ and $l\left(Q_{i}^{k+1}\right)<\alpha l\left(Q_{j}^{k}\right)$

2) $\sum\left(l\left(Q_{i}^{k+1}\right)\right)^{d} \geq \beta\left(l\left(Q_{j}^{k}\right)\right)^{d}$, where the sum is taken over all cubes $Q_{i}^{k+1}$ such that $Q_{i}^{k+1} \subset Q_{j}^{k}$.

Then

$$
\operatorname{Dim}\left(\bigcap_{k=0}^{\infty} E_{k}\right) \geq \frac{\log \left(\beta / \alpha^{d}\right)}{\log (1 / \alpha)} .
$$

In Sections 3 and 4 we will show that, under certain assumptions, $\mathcal{D}(\nabla F)$ contains Cantor-like subsets of Hausdorff dimension arbitrarily close to $d$. 
Pick an integer $N \geq 2$ that will remain fixed throughout the section. Let $Q \subset \mathbb{R}^{d}$ be a cube and let $l=l(Q)$. Divide each side of $Q$ into $N$ open-closed intervals of length $l / N$. In this way we get $N^{d}$ disjoint cubes of sidelength $l / N$ whose union is $Q$. We call them the $N$-adic descendants of $Q$ of the first generation. When repeating this to each descendant of a first generation cube we get $N^{2 d}$ disjoint cubes of sidelength $l / N^{2}$ whose union is $Q$, the $N$-adic descendants of $Q$ of the second generation. We denote by $\mathcal{E}_{j}(Q)$ the family of the $N^{j d}$ descendent cubes of $Q$ of generation $j$. If $Q_{j} \in \mathcal{E}_{j}(Q)$ then there is a chain $Q=Q_{0} \supset Q_{1} \supset \cdots \supset Q_{j}$ where $Q_{i} \in \mathcal{E}_{i}(Q), i=1, \ldots, j$. We call it the $N$-adic tower from $Q_{j}$ to $Q$. Finally, let $\mathcal{E}(Q)=\bigcup_{j=0}^{\infty} \mathcal{E}_{j}(Q)$ be the family of all $N$-adic subcubes of $Q$. The reason for using $N$-adic divisions instead of just dyadic divisions is merely technical.

Let $F$ be a harmonic function in $\mathbb{R}^{d+1}$ such that $\nabla F \in \mathcal{B}\left(\mathbb{R}_{+}^{d+1}\right)$. We describe now a stopping-time argument that will produce a Cantor-like set contained in $\mathcal{D}(\nabla F)$. For cube $Q \subset \mathbb{R}^{d}$ of sidelength $l(Q)$, we hereafter write

$$
\widehat{Q}=Q \times[0, l(Q)] \text { and }(\nabla F)_{Q}=\frac{1}{m_{d}(Q)} \int_{Q} \nabla F(x, l(Q)) d x,
$$

where the integral is understood in a vector-valued sense.

Fix a cube $Q \subset \mathbb{R}^{d}$. For any large positive number $M$ we define a family $\mathcal{S}_{M}(Q) \subset \mathcal{E}(Q)$ of $N$-adic subcubes of $Q$ in the following way. Given $Q_{j} \in \mathcal{E}_{j}(Q)$ whose $N$-adic tower is denoted by $Q=Q_{0} \supset Q_{1} \supset \cdots \supset Q_{j}$, we say that $Q_{j} \in$ $\mathcal{S}_{M}(Q)$ if and only if

$$
\left|(\nabla F)_{Q}-(\nabla F)_{Q_{i}}\right| \leq M, \quad i=1, \ldots, j-1,
$$

and

$$
\left|(\nabla F)_{Q}-(\nabla F)_{Q_{j}}\right|>M .
$$

In other words, the family $\mathcal{S}_{M}(Q)$ consists of the maximal $N$-adic subcubes $Q^{\prime}$ of $Q$ which satisfy $\left|(\nabla F)_{Q}-(\nabla F)_{Q_{j}}\right|>M$. The following proposition collects the main facts about $\mathcal{S}_{M}(Q)$.

Proposition 3.3. Let $F$ be harmonic in $\mathbb{R}_{+}^{d+1}$ and such that $\nabla F \in \mathcal{B}\left(\mathbb{R}_{+}^{d+1}\right)$. Assume that $m_{d}(\mathcal{D}(\nabla F))=0$. Then there exists a constant $C=C(N, d)>0$ such that:

1) For each $Q^{\prime} \in \mathcal{S}_{M}(Q)$, we have

$$
M<\left|(\nabla F)_{Q}-(\nabla F)_{Q^{\prime}}\right| \leq M+C\|\nabla F\|_{\mathcal{B}} .
$$

Furthermore, if $x \in Q^{\prime}$, and $l\left(Q^{\prime}\right) \leq y \leq l(Q)$, then

$$
\left|\nabla F(x, y)-(\nabla F)_{Q}\right| \leq M+C\|\nabla F\|_{\mathcal{B}} .
$$

2) $m_{d}\left(Q \backslash \bigcup_{\mathcal{S}_{M}(Q)} Q^{\prime}\right)=0$

3) Each $Q^{\prime} \in \mathcal{S}_{M}(Q)$ is a $N$-adic subcube of $Q$ of generation at least $\frac{M}{C\|\nabla F\|_{\mathcal{B}}}$. 
Proof. Part 1) follows from Proposition 2.1. Part 2) is consequence of part 1) and the assumption that $\mathcal{D}(\nabla F)$ has zero Lebesgue measure. To show 3), let $Q=Q_{0} \supset Q_{1} \supset \cdots \supset Q_{j}=Q^{\prime}$ be the $N$-adic tower from $Q^{\prime}$ to $Q$. Then

$$
M<\left|(\nabla F)_{Q}-(\nabla F)_{Q^{\prime}}\right| \leq \sum_{i=1}^{j}\left|(\nabla F)_{Q_{i-1}}-(\nabla F)_{Q_{i}}\right| \leq C j\|\nabla F\|_{\mathcal{B}} .
$$

Given $a \in \mathbb{R}^{d+1} \backslash\{0\}$ and $0<\theta<\pi / 2$, let

$$
\Gamma_{\theta}(a)=\left\{b \in \mathbb{R}^{d+1}:(a-b) \cdot a>|a||a-b| \cos \theta\right\}
$$

be the symmetric cone of vertex $a$ and aperture $2 \theta$, whose axis is the line containing the origin and the point $a$. Given $M>0$ and $0<\theta<\pi / 2$, we introduce a subfamily of $\mathcal{S}_{M}(Q)$, denoted by $\mathcal{S}_{M, \theta}(Q)$, that will play a role later. First, if $(\nabla F)_{Q}=0$, we take $\mathcal{S}_{M, \theta}(Q)=\mathcal{S}_{M}(Q)$. If $(\nabla F)_{Q} \neq 0$, let $\xi_{Q}=-(\nabla F)_{Q} /\left|(\nabla F)_{Q}\right|$ and define $\mathcal{S}_{M, \theta}(Q)$ to be the collection of all cubes $Q^{\prime} \in \mathcal{S}_{M}(Q)$ that satisfy

$$
\left((\nabla F)_{Q}-(\nabla F)_{Q^{\prime}}\right) \cdot \xi_{Q}>\cos \theta\left|\left((\nabla F)_{Q}-(\nabla F)_{Q^{\prime}}\right)\right| .
$$

Observe that if $(\nabla F)_{Q} \neq 0$, then $\mathcal{S}_{M, \theta}(Q)$ consists exactly of those cubes $Q^{\prime} \in$ $\mathcal{S}_{M}(Q)$ for which $(\nabla F)_{Q^{\prime}} \in \Gamma_{\theta}\left((\nabla F)_{Q}\right)$.

We will need the following elementary lemmas.

Lemma 3.4. Fix $\pi / 3 \leq \theta<\pi / 2, R \geq 0$ and $\kappa \geq 0$ such that $R \geq \kappa / \cos \theta$. Let $a, b \in \mathbb{R}^{d+1}$ be such that $b \in \Gamma_{\theta}(a)$ if $a \neq 0$. Suppose that $R \cos \theta \leq|a-b| \leq$ $R \cos \theta+\kappa$. Then

$$
|a| \leq R \Longrightarrow|b| \leq \sqrt{R^{2} \sin ^{2} \theta+\kappa^{2}} \leq R .
$$

Proof. From the cosine theorem and the assumption $b \in \Gamma_{\theta}(a)$ it is enough to compute the maximum of of $g(x, y)=\left(x^{2}+y^{2}-2 x y \cos \theta\right)^{1 / 2}$ in the rectangle $[0, R] \times[R \cos \theta, R \cos \theta+\kappa]$. It is easy to check that the maximum must be attained at one of the two corners $(0, R \cos \theta+\kappa)$ or $(R, R \cos \theta+\kappa)$ and that, $g(0, R \cos \theta+$ $\kappa) \leq g(R, R \cos \theta+\kappa)=\sqrt{R^{2} \sin ^{2} \theta+\kappa^{2}}$ provided $R \geq \kappa / \cos \theta, \pi / 3 \leq \theta<\pi / 2$. This proves the lemma.

The following refinement of Lemma 3.4 will be needed to deal with the little Bloch case.

Lemma 3.5. Let $\pi / 3 \leq \theta<\pi / 2$, let $\left\{\kappa_{n}\right\}$ be a bounded sequence of positive numbers, and let $g(x)=\sqrt{x(x+1)}$ for $x \geq 0$. Let $R_{1} \geq g\left(\kappa_{1} / \cos \theta\right)$ and define recursively a sequence of positive numbers $R_{n}, n=1,2 \ldots$, by

$$
R_{n+1}=\max \left\{g\left(\frac{\kappa_{n+1}}{\cos \theta}\right), \sqrt{R_{n}^{2} \sin ^{2} \theta+\kappa_{n}^{2}}\right\} .
$$

Then

$$
\limsup _{n} R_{n}=g\left((\cos \theta)^{-1} \lim \sup _{n} \kappa_{n}\right) .
$$

In particular, if $\kappa_{n} \rightarrow 0$ then $R_{n} \rightarrow 0$ but $R_{n} / \kappa_{n} \rightarrow \infty$. 
Proof. Let $\kappa=\limsup \kappa_{n} \kappa_{n}$ and $R=\limsup _{n} R_{n}$. From the construction of $R_{n}$ we have that $\left\{R_{n}\right\}$ is bounded and $R_{n} \geq g\left(\kappa_{n} / \cos \theta\right)$. Hence $R \geq g(\kappa / \cos \theta)$. On the other hand,

$$
R=\max \left\{g\left(\frac{\kappa}{\cos \theta}\right), \sqrt{R^{2} \sin ^{2} \theta+\kappa^{2}}\right\},
$$

which implies $R \leq g(\kappa / \cos \theta)$, since $g(x) \geq x$ for $x \geq 0$. If $\kappa_{n} \rightarrow 0$, it follows that $R_{n} / \kappa_{n} \rightarrow \infty$ since $R_{n} \geq g\left(\kappa_{n} / \cos \theta\right)$.

Corollary 3.6. Let $R \geq 0, \pi / 3 \leq \theta<\pi / 2, M=R \cos \theta$, and $F, Q, C$ and $\mathcal{S}_{M}(Q)$ be as in Proposition 3.3. Consider the subfamily $\mathcal{S}_{M, \theta}(Q)$ defined in (3.2). Assume that $R \geq C\|\nabla F\|_{\mathcal{B}} / \cos \theta$. Then for each $Q^{\prime} \in \mathcal{S}_{M, \theta}(Q)$,

$$
\left|(\nabla F)_{Q}\right| \leq R \Rightarrow\left|(\nabla F)_{Q^{\prime}}\right| \leq \sqrt{R^{2} \sin ^{2} \theta+C^{2}\|\nabla F\|_{\mathcal{B}}^{2}} \leq R .
$$

Furthermore, if $\left|(\nabla F)_{Q}\right| \leq R$ then

$$
|\nabla F(x, y)| \leq(1+\cos \theta) R+C\|\nabla F\|_{\mathcal{B}}
$$

whenever $x \in Q^{\prime}$ and $l\left(Q^{\prime}\right) \leq y \leq l(Q)$.

Proof. Take $a=(\nabla F)_{Q}, b=(\nabla F)_{Q^{\prime}}$ and $\kappa=C\|\nabla F\|_{\mathcal{B}}$. By Proposition 3.3, $R \cos \theta \leq|a-b| \leq R \cos \theta+\kappa$. Since $R \geq \kappa / \cos \theta$, (3.5) follows from Lemma 3.4. Inequality (3.6) also follows from part 1) of Proposition 3.3.

Now we are ready to construct a Cantor-type set that will be contained in $\mathcal{D}(\nabla F)$. Start with a fixed cube $Q_{0} \subset \mathbb{R}^{d}$. Fix $\pi / 3 \leq \theta<\pi / 2$ and $M>0$. For each $k \geq 0$ we will define a family of cubes $\mathcal{G}_{k}$ as follows. Let $\mathcal{G}_{0}=\left\{Q_{0}\right\}$ and $\mathcal{G}_{1}=\mathcal{S}_{M}\left(Q_{0}\right)$. If $k \geq 2$ and $\mathcal{G}_{k-1}$ has already been defined, we define

$$
\mathcal{G}_{k}=\bigcup_{Q \in \mathcal{G}_{k-1}} \mathcal{S}_{M, \theta}(Q) .
$$

Let

$$
E_{k}=\bigcup_{Q \in \mathcal{G}_{k}} Q \text { and } E_{\infty}=\bigcap_{k=0}^{\infty} E_{k}
$$

Observe that from the construction and Proposition 3.3, assumption 1) of Lemma 3.2 is satisfied with $\alpha=N^{-M /\left(C\|\nabla F\|_{\mathcal{B}}\right)}$.

Proposition 3.7. Fix $\pi / 3 \leq \theta<\pi / 2, R>0$ and $M=R \cos \theta$. Let $F$ and $C$ be as in Proposition 3.3. Given a cube $Q_{0} \subset \mathbb{R}^{d}$, construct $E_{\infty}$ as in (3.7). Suppose that

$$
R \geq \max \left\{\frac{C\|\nabla F\|_{\mathcal{B}}}{\cos \theta},\left|(\nabla F)_{Q_{0}}\right|\right\}
$$

Then

$$
E_{\infty} \subset\left\{x \in Q_{0}: \sup _{0<y \leq l\left(Q_{0}\right)}|\nabla F(x, y)| \leq 2 R\right\}
$$


Proof. First observe that from (3.5) in Corollary 3.6, we deduce that if $k \geq 0$ and $Q \in \mathcal{G}_{k}$ then $\left|(\nabla F)_{Q}\right| \leq R$. Therefore, from Corollary 3.6 and the assumption on $\theta$ we get

$$
|\nabla F(x, y)| \leq(1+\cos \theta) R+C\|\nabla F\|_{\mathcal{B}} \leq 2 R
$$

if $x \in E_{\infty}$ and $0<y \leq l\left(Q_{0}\right)$.

Corollary 3.8. Let $F$ be a harmonic function in $\mathbb{R}_{+}^{d+1}$ such that $\nabla F \in \mathcal{B}\left(\mathbb{R}_{+}^{d+1}\right)$. Fix $\pi / 3 \leq \theta<\pi / 2, R>0$ and $M=R \cos \theta$ and let $C>0$ be the constant appearing in Proposition 3.3. Suppose that there is a constant $0<\beta<1$ such that for any cube $Q_{0} \subset \mathbb{R}^{d}$, any subcube $Q \subset Q_{0}$, and any $R$ satisfying

$$
R \geq \max \left\{\frac{2 C\|\nabla F\|_{\mathcal{B}}}{\cos \theta},\left|(\nabla F)_{Q_{0}}\right|, \frac{C\|\nabla F\|_{\mathcal{B}} \log (1 / \beta)}{d \log N}\right\},
$$

we have

$$
\sum_{Q^{\prime} \in \mathcal{S}_{M, \theta}(Q)}\left(l\left(Q^{\prime}\right)\right)^{d} \geq \beta(l(Q))^{d} .
$$

Then

$$
\operatorname{Dim}\left\{x \in Q_{0}: \sup _{0<y \leq l\left(Q_{0}\right)}|\nabla F(x, y)| \leq 2 R\right\} \geq d-\frac{C\|\nabla F\|_{\mathcal{B}} \log (1 / \beta)}{R(\cos \theta) \log N} .
$$

In particular,

for any cube $Q_{0} \subset \mathbb{R}^{d}$.

$$
\operatorname{Dim}\left(\mathcal{D}(\nabla F) \cap Q_{0}\right)=d
$$

Proof. If $\beta$ is as in (3.9) and $\alpha=N^{-M /\left(C\|\nabla F\|_{\mathcal{B}}\right)}$, the result follows from Proposition 3.7 and Lemma 3.2.

\section{A weak quasiregularity condition for harmonic gradients}

Let $F: \mathbb{R}_{+}^{d+1} \rightarrow \mathbb{R}$ be harmonic such that $\nabla F$ is a Bloch vector field. The aim of this section is to ascertain conditions on $F$ implying that the homogeneity condition (3.9) in the stopping-time construction in Section 3 holds. By Corollary 3.8, this would imply that $\operatorname{Dim}(\mathcal{D}(\nabla F))=d$ and therefore it would essentially prove part 1) in Theorem 1 (see Section 5 for details). We will see in this section that one such condition is what we call weak quasiregularity of $\nabla F$. The section is then devoted to showing that if $\nabla F$ satisfies a weak quasiregularity condition then the technical homogeneity condition (3.9) is satisfied (see Corollary 4.5 below).

We recall some well known facts from elementary linear algebra. Suppose that $A=\left(a_{i j}\right)$ is a $(d+1) \times(d+1)$ symmetric matrix. Then

$$
\min \left|\lambda_{i}\right|=\min _{|e|=1}|A e| \leq \max _{|e|=1}|A e|=\max \left|\lambda_{i}\right|,
$$

where $\left\{\lambda_{i}\right\}$ are the eigenvalues of $A$. Therefore, $A$ distorts exactly in the same way along all directions if and only if $A$ is a conformal matrix, that is, all its eigenvalues have the same absolute value. 
On the other hand, observe that

$$
\|A\|^{2}=\sum_{i, j=1}^{d+1} a_{i j}^{2}=\sum_{i=1}^{d+1}\left|A e_{i}\right|^{2},
$$

where $e_{1}, \ldots, e_{d+1}$ is the canonical basis of $\mathbb{R}^{d+1}$. Furthermore,

$$
\frac{1}{\sqrt{d+1}}\|A\| \leq \max _{|e|=1}|A e| \leq\|A \mid\| \text {. }
$$

If $F: \mathbb{R}_{+}^{d+1} \rightarrow \mathbb{R}$, let $H F$ be the Hessian matrix of $F$, that is, the $(d+1) \times(d+1)$ matrix of all second derivatives of $F$. If $\xi \in \mathbb{R}^{d+1}$ we interpret $(H F) \xi$ as the function obtained by matrix multiplication. Furthermore, in accord to the previous comments we write

$$
\|H F(x, y)\|^{2}=\sum_{i, j=1}^{d+1}\left|\frac{\partial^{2} F}{\partial x_{i} \partial x_{j}}(x, y)\right|^{2}
$$

Here we write $x=\left(x_{1}, \ldots, x_{d}\right) \in \mathbb{R}^{d}$ and $x_{d+1}=y$. Fix $0<\delta<1$. For each cube $Q \subset \mathbb{R}^{d}$ we define the $\delta$-hyperbolic box associated to $Q$ by

$$
C_{\delta}(Q)=\left\{(x, y) \in \mathbb{R}_{+}^{d+1}: x \in Q, \delta l(Q) \leq y \leq l(Q)\right\} .
$$

Let $0<\delta<1 \leq \gamma$. We say that $\nabla F$ satisfies a $\delta$ - weak quasiregularity condition with constant $\gamma$ if for any cube $Q \subset \mathbb{R}^{d}$ there holds the inequality

$$
\int_{C_{\delta}(Q)} \max _{|e|=1}|(H F) e(x, y)|^{2} d x d y \leq \gamma^{2} \min _{|e|=1} \int_{C_{\delta}(Q)}|(H F) e(x, y)|^{2} d x d y,
$$

where the max and min are taken over all unitary vectors $e \in \mathbb{R}^{d+1}$. If we are not interested in the particular value of the constant $\gamma$ we will just say that $\nabla F$ satisfies a $\delta$-weak QR condition. For technical reasons in this section we will only use values of $\delta$ of the form $1 / N$ for an integer $N \geq 2$.

Now fix an integer $N \geq 2$, a sufficiently large number $M>0$, and a cube $Q \subset \mathbb{R}^{d}$. Let $\mathcal{S}_{M}(Q)$ be the family of $N$-adic subcubes of $Q$ introduced in Section 3 . For $j \geq 1$, let $\mathcal{S}_{M}^{j}(Q)$ be the family of all cubes $Q^{\prime} \in \mathcal{S}_{M}(Q)$ of generation at most $j$. Define also

$$
\begin{aligned}
T(Q) & =\{(x, l(Q)): x \in Q\}, \\
T_{j}(Q) & =\bigcup_{Q^{\prime} \in \mathcal{S}_{M}^{j}(Q)} T\left(Q^{\prime}\right), \quad S_{j}(Q)=\bigcup_{Q^{\prime} \in \mathcal{S}_{M}^{j}(Q)} Q^{\prime}, \\
B_{j}(Q) & =\left\{\left(x, N^{-j} l(Q)\right): x \in Q \backslash S_{j}(Q)\right\}, \\
\Omega_{j}(Q) & =\left(\widehat{Q} \backslash \bigcup_{Q^{\prime} \in \mathcal{S}_{M}^{j}(Q)} \widehat{Q^{\prime}}\right) \cap\left\{(x, y): x \in Q, N^{-j} l(Q)<y<l(Q)\right\} .
\end{aligned}
$$


Note that $\Omega_{j}(Q)$ is a sort of truncated domain associated to the stopping time originating $\mathcal{S}_{M}(Q)$. In order to show that the cubes in $\mathcal{S}_{M, \theta}(Q)$ take a fixed amount of the $d$-dimensional measure of $Q$ (as required in assumption (3.9)) we will need to assume that $\nabla F$ satisfies a QR condition. The technique basically consists of using Green's formula applied to the functions $y$ and $\left|\nabla F-(\nabla F)_{Q}\right|^{2}$ in the domains $\Omega_{j}$. We will prove a sequence of technical lemmas. In the rest of the section, $m_{d}(E)$ stands for the $d$-dimensional Lebesgue measure of $E \subset \mathbb{R}^{d}$.

Lemma 4.1. Let $F$ be a harmonic function in $\mathbb{R}_{+}^{d+1}$ such that $\nabla F \in \mathcal{B}\left(\mathbb{R}_{+}^{d+1}\right)$ and $m_{d}(\mathcal{D}(\nabla F))=0$. Let $M>0, Q$, and $\Omega_{j}(Q)$ be as above. Then there is a constant $C=C(d, N)>0$ such that if $M>C\|\nabla F\|_{\mathcal{B}}$, then

$$
\int_{\Omega_{j}(Q)} y\|H F(x, y)\|^{2} d x d y \geq \frac{1}{8} M^{2}(l(Q))^{d}
$$

for sufficiently large $j$.

Proof. Note that $\Omega_{j}=\Omega_{j}(Q)$ is bounded by a finite number of smooth hypersurfaces. We apply Green's formula to $y$ and $\mid\left(\nabla F-\left.(\nabla F)_{Q}\right|^{2}\right.$ in $\Omega_{j}$. By simple computation,

$$
\begin{aligned}
& \nabla\left(\mid\left(\nabla F-\left.(\nabla F)_{Q}\right|^{2}\right)=2(H F)\left(\nabla F-(\nabla F)_{Q}\right),\right. \\
& \triangle\left(\mid\left(\nabla F-\left.(\nabla F)_{Q}\right|^{2}\right)=2\|H F\|^{2} .\right.
\end{aligned}
$$

Hence, from Green's formula, the integral in (4.3) is equal to

$$
\int_{\partial \Omega_{j}} y(H F)\left(\nabla F-(\nabla F)_{Q}\right) \cdot n-\frac{1}{2} \int_{\partial \Omega_{j}}\left|\nabla F-(\nabla F)_{Q}\right|^{2} \nabla(y) \cdot n,
$$

where $n$ denotes the outer normal unit vector. Observe first that by construction of $\Omega_{j}$ and Proposition 3.3, we have

$$
\begin{aligned}
& \left|\nabla F-(\nabla F)_{Q}\right| \leq M+C\|\nabla F\|_{\mathcal{B}} \quad \text { on } \Omega_{j}, \\
& \left|\nabla F-(\nabla F)_{Q}\right| \geq M-C\|\nabla F\|_{\mathcal{B}} \quad \text { on } \bigcup_{Q^{\prime} \in \mathcal{S}_{M}^{j}(Q)} T\left(Q^{\prime}\right),
\end{aligned}
$$

with $C=C(d, N)$. From the Bloch condition, (4.5), and the fact that the surface measure of $\partial \Omega_{j}$ is smaller than a fixed multiple of $(l(Q))^{d}$, it follows that

$$
\left|\int_{\partial \Omega_{j}} y(H F)\left(\nabla F-(\nabla F)_{Q}\right) \cdot n\right| \leq C\|\nabla F\|_{\mathcal{B}}\left(M+C\|\nabla F\|_{\mathcal{B}}\right)(l(Q))^{d},
$$

where $C=C(d, N)$. On the other hand,

$$
\int_{\partial \Omega_{j}}\left|\nabla F-(\nabla F)_{Q}\right|^{2} \nabla(y) \cdot n=\int_{T(Q)}\left|\nabla F-(\nabla F)_{Q}\right|^{2}-\int_{T_{j}(Q) \cup B_{j}(Q)}\left|\nabla F-(\nabla F)_{Q}\right|^{2} .
$$


From the Bloch condition we get

$$
\begin{aligned}
& \int_{T(Q)}\left|\nabla F-(\nabla F)_{Q}\right|^{2} \leq\left(C\|\nabla F\|_{\mathcal{B}}\right)^{2}(l(Q))^{d}, \\
& \int_{B_{j}(Q)}\left|\nabla F-(\nabla F)_{Q}\right|^{2} \leq\left(M+C\|\nabla F\|_{\mathcal{B}}\right)^{2}\left((l(Q))^{d}-m_{d}\left(S_{j}(Q)\right)\right) .
\end{aligned}
$$

On the other hand, from (4.6) we get

$$
\int_{T_{j}(Q)}\left|\nabla F-(\nabla F)_{Q}\right|^{2} \geq\left(M-C\|\nabla F\|_{\mathcal{B}}\right)^{2} m_{d}\left(S_{j}(Q)\right) .
$$

Now, by part 2) of Proposition 3.3 we have that $m_{d}\left(S_{j}(Q)\right) \rightarrow(l(Q))^{d}$ as $j \rightarrow \infty$. Choose $j$ large enough so that $m_{d}\left(S_{j}(Q)\right)>\frac{3}{4}(l(Q))^{d}$. It then follows, from (4.7)-(4.9) and simple computation, that there is a positive constant $C=C(d, N)$ such that

$$
\begin{aligned}
\left|\int_{\partial \Omega_{j}}\right| \nabla F-\left.(\nabla F)_{Q}\right|^{2} \nabla(y) \cdot n \mid & \geq\left(\frac{M^{2}}{2}-3 C\|\nabla F\|_{\mathcal{B}} M-\frac{3}{2} C^{2}\|\nabla F\|_{\mathcal{B}}^{2}\right)(l(Q))^{d} \\
& \geq \frac{M^{2}}{4}(l(Q))^{d},
\end{aligned}
$$

where the last inequality holds as soon as $M>14 C\|\nabla F\|_{\mathcal{B}}$. This proves the lemma.

We need now a variant of Lemma 4.1.

Lemma 4.2. Let $F, Q, M$ and $\Omega_{j}(Q)$ be as in Lemma 4.1. Assume that $\nabla F$ satisfies a weak $(1 / N)-Q R$ condition with constant $\gamma \geq 1$ for some integer $N \geq 2$. Then there is a constant $C=C(d, N)>0$ such that if $M>36 \gamma^{2} C\|\nabla u\|_{\mathcal{B}}$ then, for sufficiently large $j$,

$$
\min _{|e|=1} \int_{T_{j}(Q)}\left[\left(\nabla F-(\nabla F)_{Q}\right) \cdot e\right]^{2} \geq \frac{M^{2}}{16 \gamma^{2}}(l(Q))^{d},
$$

where the minimum is taken over all unitary vectors $e \in \mathbb{R}^{d+1}$.

Proof. The proof mimics the proof of Lemma 4.1. Fix a unitary vector $e \in \mathbb{R}^{d+1}$. Consider the harmonic function $v=\left(\nabla F-(\nabla F)_{Q}\right) \cdot e$. We apply Green's formula in $\Omega_{j}=\Omega_{j}(Q)$ to the functions $y$ and $v^{2}$. A simple computation shows

$$
\nabla v^{2}=2\left(\left(\nabla F-(\nabla F)_{Q}\right) \cdot e\right)(H F) e \quad \text { and } \quad \triangle\left(v^{2}\right)=2|(H F) e|^{2} .
$$

Therefore, as in Lemma 4.1,

$$
\begin{aligned}
& \int_{\Omega_{j}} 2 y|(H F) e|^{2} \\
& \quad=\int_{\partial \Omega_{j}} 2 y\left(\nabla F-(\nabla F)_{Q} \cdot e\right)(H F) e \cdot n-\int_{\partial \Omega_{j}}\left[\left(\nabla F-(\nabla F)_{Q}\right) \cdot e\right]^{2} \nabla(y) \cdot n .
\end{aligned}
$$


Now observe that, since $\Omega_{j}$ can be decomposed as a union of disjoint hyperbolic boxes of the form $C_{1 / N}\left(Q^{\prime}\right)$, we have, from the $1 / N$-QR condition and Lemma 4.1,

$$
\frac{M^{2}}{8}(l(Q))^{d} \leq \int_{\Omega_{j}} y\|H F\|^{2} \leq 2 \gamma^{2} \int_{\Omega_{j}} y|(H F) e|^{2} .
$$

On the other hand, as in Lemma 4.1,

$$
\left|\int_{\partial \Omega_{j}} y\left(\nabla F-(\nabla F)_{Q}\right) \cdot e(H F) e \cdot n\right| \leq C\|\nabla F\|_{\mathcal{B}}\left(M+C\|\nabla F\|_{\mathcal{B}}\right)(l(Q))^{d}
$$

As for the other surface integral, notice that $\nabla(y) \cdot n$ vanishes outside the horizontal part of $\partial \Omega_{j}$, which consists of $T_{j}(Q) \cup T(Q) \cup B_{j}(Q)$. Furthermore,

$$
\begin{aligned}
& \int_{T(Q)}\left[\left(\nabla F-(\nabla F)_{Q}\right) \cdot e\right]^{2} \leq C^{2}\|\nabla F\|_{\mathcal{B}}^{2}(l(Q))^{d}, \\
& \int_{B_{j}(Q)}\left[\left(\nabla F-(\nabla F)_{Q}\right) \cdot e\right]^{2} \leq\left(M+C\|\nabla F\|_{\mathcal{B}}\right)^{2} m_{d}\left(B_{j}(Q)\right) .
\end{aligned}
$$

If $j$ is large enough, $m_{d}\left(B_{j}(Q)\right)$ can be made arbitrarily small. Then, combining (4.11)-(4.14), we finally get

$$
\int_{T_{j}(Q)}\left[\left(\nabla F-(\nabla F)_{Q}\right) \cdot e\right]^{2} \geq \frac{M^{2}}{16 \gamma^{2}}(l(Q))^{d}
$$

as soon as $M>36 \gamma^{2} C\|\nabla F\|_{\mathcal{B}}$.

Let $0<\theta<\pi / 2$ and $e \in \mathbb{R}^{d+1}$ be a unitary vector. Define

$$
\begin{aligned}
& \mathcal{S}_{M, e, \theta}^{j,+}(Q)=\left\{Q^{\prime} \in \mathcal{S}_{M}^{j}(Q):\left((\nabla F)_{Q^{\prime}}-(\nabla F)_{Q}\right) \cdot e>\cos \theta\left|(\nabla F)_{Q^{\prime}}-(\nabla F)_{Q}\right|\right\}, \\
& \mathcal{S}_{M, e, \theta}^{j,-}(Q)=\left\{Q^{\prime} \in \mathcal{S}_{M}^{j}(Q):\left((\nabla F)_{Q^{\prime}}-(\nabla F)_{Q}\right) \cdot e<-\cos \theta\left|(\nabla F)_{Q^{\prime}}-(\nabla F)_{Q}\right|\right\}, \\
& \mathcal{S}_{M, e, \theta}^{j}(Q)=\mathcal{S}_{M, e, \theta}^{j,+}(Q) \cup \mathcal{S}_{M, e, \theta}^{j,-}(Q) .
\end{aligned}
$$

If $(\nabla F)_{Q} \neq 0$ and we make the particular choice $e=-(\nabla F)_{Q} /\left|(\nabla F)_{Q}\right|$ we recover the family

$$
\mathcal{S}_{M, \theta}(Q)=\bigcup_{j} \mathcal{S}_{M, e, \theta}^{j,+}(Q)
$$

introduced in Section 3.

Lemma 4.3. Assume that $\nabla F$ satisfies a weak $1 / N-Q R$ condition with constant $\gamma \geq 1$. There is a constant $C=C(d, N)>0$ such that if $M>36 \gamma^{2} C\|\nabla F\|_{\mathcal{B}}$ and $\cos \theta \leq 1 / 8 \gamma$ then

$$
\sum_{Q^{\prime} \in \mathcal{S}_{M, e, \theta}^{j}(Q)}\left(l\left(Q^{\prime}\right)\right)^{d} \geq \frac{1}{150 \gamma^{2}}(l(Q))^{d}
$$

for any unitary vector $e \in \mathbb{R}^{d+1}$. 
Proof. If $Q^{\prime} \in \mathcal{S}_{M}^{j}(Q) \backslash \mathcal{S}_{M, e, \theta}^{j}(Q)$ then on $T\left(Q^{\prime}\right)$ we have the estimate

$$
\left|\left(\nabla F-(\nabla F)_{Q}\right) \cdot e\right| \leq C\|\nabla F\|_{\mathcal{B}}+\cos \theta\left(M+C\|\nabla F\|_{\mathcal{B}}\right),
$$

while, if $Q^{\prime} \in \mathcal{S}_{M, e, \theta}^{j}(Q)$ then on $T\left(Q^{\prime}\right)$,

$$
\left|\left(\nabla F-(\nabla F)_{Q}\right) \cdot e\right| \leq M+C\|\nabla F\|_{\mathcal{B}} .
$$

Therefore

$$
\begin{aligned}
\int_{T_{j}(Q)}\left[\left(\nabla F-(\nabla F)_{Q}\right) \cdot e\right]^{2} \leq & {\left[C\|\nabla F\|_{\mathcal{B}}+\cos \theta\left(M+C\|\nabla F\|_{\mathcal{B}}\right)\right]^{2}(l(Q))^{d} } \\
& +\left(M+C\|\nabla F\|_{\mathcal{B})^{2}} \sum_{Q^{\prime} \in \mathcal{S}_{M, e, \theta}^{j}(Q)}\left(l\left(Q^{\prime}\right)\right)^{d}\right.
\end{aligned}
$$

and from Lemma 4.2 we obtain

$$
\begin{aligned}
\sum_{Q^{\prime} \in \mathcal{S}_{M, e, \theta}^{j}(Q)}\left(l\left(Q^{\prime}\right)\right)^{d} & \geq \frac{1 /\left(16 \gamma^{2}\right)-\left[\cos \theta+(1+\cos \theta) C\|\nabla F\|_{\mathcal{B} / M}\right]^{2}}{\left(1+C\|\nabla F\|_{\mathcal{B}} / M\right)^{2}}(l(Q))^{d} \\
& \geq \frac{1}{150 \gamma^{2}}(l(Q))^{d}
\end{aligned}
$$

provided $M>32 \gamma C\|\nabla F\|_{\mathcal{B}}$ and $\cos \theta \leq 1 /(8 \gamma)$.

Lemma 4.4. Let $N, F, Q, M$, and $\Omega_{j}$ as in Lemma 4.1. Suppose that $\nabla F$ satisfies a weak $1 / N-Q R$ condition with constant $\gamma \geq 1$. Let $0<\theta<\pi / 2$ be such that $\cos \theta \leq 1 / 6400 \gamma^{3}$. There is a constant $C=C(d, N)>0$ such that for any unitary vector $e \in \mathbb{R}^{d+1}$ and sufficiently large $j$ we have

$$
\sum_{Q^{\prime} \in \mathcal{S}_{M, e, \theta}^{j,+}(Q)}\left(l\left(Q^{\prime}\right)\right)^{d} \geq \frac{(l(Q))^{d}}{16000 \gamma^{3}}
$$

provided $M>10^{5} \gamma^{3} C\|\nabla F\|_{\mathcal{B}}$.

Proof. Fix $M$ and $e$. Choose $0<\theta_{1}<\theta_{2}=\theta$ such that $\cos \theta_{1}=1 /(8 \gamma)$. For $i=1,2$, let $\mathcal{A}_{i}^{+}=\mathcal{S}_{M, e, \theta_{i}}^{j,+}(Q), \mathcal{A}_{i}^{-}=\mathcal{S}_{M, e, \theta_{i}}^{j,-}(Q)$ and $\mathcal{A}_{i}=\mathcal{A}_{i}^{+} \cup \mathcal{A}_{i}^{-}$. Furthermore, set

$$
A_{i}^{+}=\bigcup_{Q^{\prime} \in \mathcal{A}_{i}^{+}} T\left(Q^{\prime}\right), \quad A_{i}^{-}=\bigcup_{Q^{\prime} \in \mathcal{A}_{i}^{-}} T\left(Q^{\prime}\right),
$$

and $A_{i}=A_{i}^{+} \cup A_{i}^{-}$and $H_{i}=T_{j}(Q) \backslash A_{i}$. From Lemma 4.3, we have $m_{d}\left(A_{1}\right)=$ $m_{d}\left(A_{1}^{+}\right)+m_{d}\left(A_{1}^{-}\right) \geq \frac{1}{150 \gamma^{2}}(l(Q))^{d}$. If $m_{d}\left(A_{1}^{+}\right) \geq \frac{1}{300 \gamma^{2}}(l(Q))^{d}$ we are done, so assume that $m_{d}\left(A_{1}^{-}\right) \geq \frac{1}{300 \gamma^{2}}(l(Q))^{d}$. Since $\left|\left((\nabla F)_{Q^{\prime}}-(\nabla F)_{Q}\right) \cdot e\right|>M \cos \theta_{1}$ for any $Q^{\prime} \in A_{1}^{-}$, we deduce that

$$
\left.\int_{A_{1}^{-}} \mid \nabla F-(\nabla F)_{Q}\right) \cdot e \mid \geq\left(\cos \theta_{1} M-C\|\nabla F\|_{\mathcal{B}}\right) m_{d}\left(A_{1}^{-}\right) \geq \frac{M(l(Q))^{d}}{3200 \gamma^{3}},
$$

where for the last inequality we have used $\cos \theta_{1}=1 /(8 \gamma)$ and $M>32 \gamma C\|\nabla F\|_{\mathcal{B}}$. 
Now we apply Green's formula in $\Omega_{j}$ to the functions $y$ and

$$
v=\left(\nabla F-(\nabla F)_{Q}\right) \cdot e .
$$

Note that $v$ is harmonic, while $\nabla v=(H F) e$. Therefore, from Green's formula,

$$
\int_{\partial \Omega_{j}}\left(\nabla F-(\nabla F)_{Q}\right) \cdot e \nabla(y) \cdot n=\int_{\partial \Omega_{j}} y(H F) e \cdot n .
$$

Again, the integrand on the left-hand side of (4.17) vanishes outside $T_{j}(Q) \cup B_{j}(Q)$ $\cup T(Q)$. Furthermore,

$$
\begin{gathered}
\left|\int_{\partial \Omega_{j}} y(H F) e \cdot n\right| \leq C\|\nabla F\|_{\mathcal{B}}(l(Q))^{d}, \\
\left|\int_{B_{j}(Q)}\left(\nabla F-(\nabla F)_{Q}\right) \cdot e\right| \leq M m_{d}\left(B_{j}(Q)\right), \\
\left|\int_{T(Q)}\left(\nabla F-(\nabla F)_{Q}\right) \cdot e\right| \leq C\|\nabla F\|_{\mathcal{B}}(l(Q))^{d} .
\end{gathered}
$$

Now we use the decomposition $T_{j}(Q)=A_{2}^{+} \cup A_{2}^{-} \cup H_{2}$ and observe that, since $A_{1}^{-} \subset A_{2}^{-}$, we get, by (4.16),

$$
\left|\int_{A_{2}^{-}}\left(\nabla F-(\nabla F)_{Q}\right) \cdot e\right| \geq \frac{M}{3200 \gamma^{3}}(l(Q))^{d} .
$$

On the other hand,

$$
\left|\int_{H_{2}}\left(\nabla F-(\nabla F)_{Q}\right) \cdot e\right| \leq\left(\cos \theta_{2}\right)\left(M+C\|\nabla F\|_{\mathcal{B}}\right)(l(Q))^{d} .
$$

Now since $m_{d}\left(B_{j}(Q)\right) \rightarrow 0$ as $j \rightarrow \infty$, we get from (4.18)-(4.22) that if $j$ is large enough,

$\int_{A_{2}^{+}}\left(\nabla F-(\nabla F)_{Q}\right) \cdot e \geq\left(M\left(\frac{1}{3200 \gamma^{3}}-\cos \theta_{2}\right)-\left(2+\cos \theta_{2}\right) C\|\nabla F\|_{\mathcal{B}}\right)(l(Q))^{d}$.

From (4.23) and the fact that $\left|\nabla F-(\nabla F)_{Q}\right| \leq M+C\|\nabla F\|_{\mathcal{B}}$ on $A_{2}^{+}$we finally deduce

$$
m_{d}\left(A_{2}^{+}\right) \geq \frac{(l(Q))^{d}}{16000 \gamma^{3}}
$$

provided $M>10^{5} \gamma^{3} C\|\nabla F\|_{\mathcal{B}}$.

Finally, we collect the previous estimates in the following statement.

Corollary 4.5. Let $F$ be a harmonic function in $\mathbb{R}_{+}^{d+1}$ such that $\nabla F \in \mathcal{B}\left(\mathbb{R}_{+}^{d+1}\right)$ and $m_{d}(\mathcal{D}(\nabla F))=0$. Assume that $\nabla F$ satisfies a weak $1 / N-Q R$ condition with constant $\gamma \geq 1$ for some integer $N \geq 2$. Then there are constants $0<\theta_{0}=\theta_{0}(\gamma)<$ $\pi / 2,0<\beta=\beta(\gamma)<1$, and $C=C(\gamma, N, d)>0$ such that for any $\theta, \theta_{0} \leq \theta<\pi / 2$, any cube $Q_{0} \subset \mathbb{R}^{d}$, any subcube $Q \subset Q_{0}$, and any $M \geq C\|\nabla F\|_{\mathcal{B}}$ we have

$$
\sum_{Q^{\prime} \in \mathcal{S}_{M, \theta}(Q)}\left(l\left(Q^{\prime}\right)\right)^{d} \geq \beta(l(Q))^{d} .
$$




\section{Proof of Theorem 1}

Proof of part 1). Fix $\pi / 3 \leq \theta<\pi / 2$ as in Corollary 4.5 and a cube $Q_{0} \subset \mathbb{R}^{d}$, and assume that $m_{d}\left(\mathcal{D}(\nabla F) \cap Q_{0}\right)=0$, because otherwise the result is trivial. If $R$ is large enough and $M=R \cos \theta$ then, by Corollary 4.5,

$$
\sum_{Q^{\prime} \in S_{M, \theta}(Q)}\left(l\left(Q^{\prime}\right)\right)^{d} \geq \beta(l(Q))^{d},
$$

where $\beta=\beta(\gamma)>0$. Now it follows from Lemma 3.2 and Corollary 3.8 that

$$
\operatorname{Dim}\left\{x \in Q_{0}: \limsup _{y \rightarrow 0}|\nabla F(x, y)| \leq 2 R\right\} \geq d-\frac{C\|\nabla F\|_{\mathcal{B}} \log (1 / \beta)}{R(\cos \theta) \log N},
$$

and the result follows upon letting $R \rightarrow \infty$.

Now we will adapt the results in previous section to cover the case of gradients in the little Bloch class (part 2) in Theorem 1). First, we obtain an analogue of Plessner's theorem on the boundary values of analytic functions in the unit disc for gradients of harmonic functions in the upper half-space which are weakly quasiregular. If $f$ is analytic in the unit disc $\mathbb{D}$, a classical result of Plessner (Theorem 6.13 in [15]) says that for almost all points $e^{i \theta} \in \partial \mathbb{D}$, either $f$ has a finite nontangential limit at $e^{i \theta}$ or the image under $f$ of any symmetric cone with vertex $e^{i \theta}$ is dense in the complex plane $\mathbb{C}$. Therefore the boundary behavior of $f$ at almost every $e^{i \theta}$ is either very good or very bad. If $z=\left(x^{\prime}, y\right) \in \mathbb{R}_{+}^{d+1}$ and $x \in \mathbb{R}^{d}$, the notation $z \varangle x$ means that $z$ tends to $x$ nontangentially, that is, $z$ tends to $x$ and

$$
z \in \Gamma_{\alpha}(x)=\left\{\left(x^{\prime}, y\right):\left|x-x^{\prime}\right| \leq y \tan \alpha, \quad 0<y<1\right\}
$$

for a fixed $0<\alpha<\pi / 2$. We refer to [17] for the main results concerning nontangential boundary behavior of harmonic functions in the upper half-space. The following proposition says that if a harmonic gradient is weakly quasiregular, then Plessner's theorem still holds. Observe that, as in the analytic case, no growth assumption is required.

Proposition 5.1. Let $u$ be harmonic in $\mathbb{R}_{+}^{d+1}$ and such that $\nabla u$ is weakly quasiregular. Then, for any cube $Q \subset \mathbb{R}^{d}$ there holds one of the two following possibilities:

1) $m_{d}\left(\left\{x \in Q: \lim _{z \varangle x} \nabla u(z)\right.\right.$ exists $\left.\}\right)>0$.

2) For any $0<\alpha<\pi / 2$ and for almost every $x \in Q, \nabla u\left(\Gamma_{\alpha}(x)\right)$ is dense in $\mathbb{R}^{d+1}$. In particular, for any $a \in \mathbb{R}^{d+1}$ and for a.e. $x \in Q$,

$$
\liminf _{z \varangle x}|\nabla u(z)-a|=0 .
$$

Proof. Fix a cube $Q \subset \mathbb{R}^{d}$. Assume that part 2) does not hold. Standard measure theoretic arguments show that there are a set $E \subset Q$ with $m_{d}(E)>0, a \in \mathbb{R}^{d+1}$, $0<\alpha_{0}<\pi / 2$, and $0<y_{0}<1$ such that

$$
\inf |\nabla u(z)-a|>0 .
$$


Here the infimum is taken over the set $\left\{z \in \mathbb{R}_{+}^{d+1}: z \in \Gamma_{\alpha_{0}}(x), x \in E\right\} \cap\{0<$ $\left.y<y_{0}\right\}$. For simplicity, $E$ may denote hereafter different subsets of $Q$ of positive $d$-dimensional measure. Choose $i \in\{1, \ldots, d+1\}$ such that

$$
\inf \left|\frac{\partial u}{\partial x_{i}}(z)-a_{i}\right|>0
$$

where, as usual, $x_{d+1}=y$ and $a=\left(a_{1}, \ldots, a_{d+1}\right)$. L. Carleson proved that a harmonic function in $\mathbb{R}_{+}^{d+1}$ which is nontangentially bounded from below on a certain set of points in $\mathbb{R}^{d}$, has a nontangential limit at almost every point of this set (see [5]). We deduce that $\lim _{z \varangle x} \partial u / \partial x_{i}(z)$ exists for almost every $x \in E$. From well-known results relating the boundary behavior and the area function of harmonic functions in the upper half-space (see Theorem 4 in Chapter VII of [5]), we deduce that

$$
A_{\alpha}^{2}\left(\frac{\partial u}{\partial x_{i}}\right)(x)=\int_{\Gamma_{\alpha}(x)} y^{1-d}\left|\nabla\left(\frac{\partial u}{\partial x_{i}}(z)\right)\right|^{2} d z<\infty
$$

for a.e $x \in E$ and any $0<\alpha<\pi / 2$. Here $A_{\alpha} v(x)$ denotes the so-called area function of $v$ associated to the aperture $\alpha$. (See [17] for details). Let $x$ and $\alpha$ be as in (5.1). Assume that $\nabla u$ satisfies a $\delta$-weak quasiregularity condition with constant $\gamma$ for a certain $0<\delta<1$. Consider the truncated cones

$$
\Gamma_{\alpha, n}(x)=\Gamma_{\alpha}(x) \cap\left\{\left(x^{\prime}, y\right): \delta^{(n+1)} \leq y \leq \delta^{n}\right\} .
$$

Then, if $Q^{\prime}$ is the cube centered at $x$ with $l\left(Q^{\prime}\right)=2 \delta^{n}$, we have

$$
\Gamma_{\beta, n}(x) \subset C_{\delta}\left(Q^{\prime}\right) \subset \Gamma_{\alpha, n}(x),
$$

where $0<\beta<\alpha$ and $\tan \beta=\delta \tan \alpha$. Therefore, since $\nabla\left(\partial u / \partial x_{i}\right)=(H u) e_{i}$, where $e_{i}$ is the $i$ th vector of the canonical basis in $\mathbb{R}^{d+1}$, we get from the $\delta$-QR condition,

$\int_{\Gamma_{\beta, n}(x)} y^{1-d}\|H u\|^{2} \leq C_{1} \gamma^{2} \int_{C_{\delta}\left(Q^{\prime}\right)} y^{1-d}\left|\nabla\left(\frac{\partial u}{\partial x_{i}}\right)\right|^{2} \leq C_{1} \gamma^{2} \int_{\Gamma_{\alpha, n}(x)} y^{1-d}\left|\nabla\left(\frac{\partial u}{\partial x_{i}}\right)\right|^{2}$,

where $C_{1}$ only depends on $\delta, \alpha$, and $d$. Therefore,

$$
A_{\beta}^{2}\left(\frac{\partial u}{\partial x_{j}}\right)(x)<\infty
$$

for all $j=1, \ldots, d+1$. Using again the results relating nontangential limits and the area function, this time in the opposite direction, we finally get that $\lim _{z \varangle x} \nabla u(z)$ exists for almost every $x \in E$. This proves the proposition.

Sketch of proof of Theorem 1, part 2).

Suppose that $F: \mathbb{R}_{+}^{d+1} \rightarrow \mathbb{R}$ is harmonic and $\nabla F \in \mathcal{B}_{0}\left(\mathbb{R}_{+}^{d+1}\right)$ satisfies a $(1 / N)$ - QR condition with constant $\gamma \geq 1$. Pick a cube $Q_{0} \subset \mathbb{R}^{d}$ and assume that $m_{d}\left(\mathcal{D}_{0}(\nabla F) \cap Q_{0}\right)=0$ since otherwise the conclusion is obvious. 
We will actually show that

$$
\operatorname{Dim}\left\{x \in Q_{0}: \lim _{y \rightarrow 0} \nabla F(x, y)=a\right\}=d
$$

for each $a \in \mathbb{R}^{d+1}$. Hence in particular $\operatorname{Dim} \mathcal{D}_{0}(\nabla F)=d$.

The proof is similar to the proof of part 1 ) of Theorem 1 , the main modifications coming from the following two key facts:

- In part 1) of Theorem 1 , we could assume that $m_{d}\left(\mathcal{D}(\nabla F) \cap Q_{0}\right)=0$, so when we ran the stopping time argument, $\nabla F$ must escape balls with full $d$-measure (see part 2) in Proposition 3.3). In part 2) of Theorem 1, the hypothesis is $m_{d}\left(\mathcal{D}_{0}(\nabla F) \cap Q_{0}\right)=0$. By Proposition 3.1, $m_{d}\left(\mathcal{D}(\nabla F) \cap Q_{0}\right)=0$. Therefore we can run the same type of stopping-time arguments using even small balls.

- Related to the previous point is the fact that the meaning of $\|\nabla F\|_{\mathcal{B}}$ should now be relaxed, in the sense that it must be understood as a variable quantity that tends to 0 as long as we approach the boundary of $\mathbb{R}_{+}^{d+1}$. This implies that $k=C\|\nabla F\|_{\mathcal{B}}$ in Sections 3 and 4 can be replaced now by a sequence $k_{n} \rightarrow 0$, where $n$ refers to the successive stopping-time steps in the construction. Consequently, it follows from Lemma 3.5 that the sequence of radius $R_{n}$ can be chosen in such a way that $R_{n} \rightarrow 0$, although $R_{n} / k_{n} \rightarrow \infty$, which allows replacing boundedness of $\nabla F$ by convergence to a given point.

A brief sketch of the proof runs as follows.

It is enough to take $a=0$ in (5.2). Fix $\pi / 3 \leq \theta<\pi / 2$ as in Corollary 4.5. Then we run a sequence of stopping times corresponding to sequences $k_{n}$ and $M_{n}=R_{n} \cos \theta$ that can be defined as follows. Suppose that $k_{j}$ and $R_{j}$ have already been chosen for $j=1, \ldots, n$. According to Proposition 3.3, part 3), we define

$$
\begin{aligned}
& k_{n+1}=\sup \left\{y\|H F(x, y)\|: 0<y \leq N^{-(\cos \theta) S_{n}} l\left(Q_{0}\right)\right\} \\
& R_{n+1}=\max \left\{g\left(\frac{k_{n+1}}{\cos \theta}\right), \sqrt{R_{n}^{2} \sin ^{2} \theta+k_{n}^{2}}\right\},
\end{aligned}
$$

where $S_{n}=\sum_{j=1}^{n} R_{j} / k_{j}$. Note that $k_{n} \rightarrow 0$ and that, by Lemma $3.5, R_{n} \rightarrow 0$ but $R_{n} / k_{n} \rightarrow \infty$. Then the same arguments as in Section 3 can be used to obtain that

$$
\operatorname{Dim}\left\{x \in Q_{0}: \lim _{y \rightarrow 0} \nabla F(x, y)=0\right\}=d
$$

\section{Some estimates for Poisson integrals}

In this section we collect some estimates relating the regularity of a function defined on $\mathbb{R}^{d}$ to the regularity of its Poisson extension to the upper half-space $\mathbb{R}_{+}^{d+1}$.

For $0<\alpha<1$ let $\Lambda_{\alpha}\left(\mathbb{R}^{d}\right)$ be the Hölder class of bounded functions $f: \mathbb{R}^{d} \rightarrow \mathbb{R}$ for which

$$
\|f\|_{\alpha}=\sup _{x, h \in \mathbb{R}^{d}} \frac{|f(x+h)-f(x)|}{\|h\|^{\alpha}}<\infty .
$$


The class $C^{1, \alpha}\left(\mathbb{R}^{d}\right)$ (resp. $C^{2, \alpha}\left(\mathbb{R}^{d}\right)$ ) consists of those differentiable functions defined in $\mathbb{R}^{d}$ whose first partial derivatives (resp. second partial derivatives) belong to the Hölder class $\Lambda_{\alpha}\left(\mathbb{R}^{d}\right)$.

The following two propositions, which relate the regularity of the Poisson extension to the regularity of the boundary data, are probably well known (see [17] for similar results in this direction). We include here a detailed proof of the first.

Proposition 6.1. Let $\phi: \mathbb{R}^{d} \rightarrow \mathbb{R}$ be such that $\phi \in C^{1, \alpha}\left(\mathbb{R}^{d}\right)$ and let $\Phi$ be the Poisson extension of $\phi$ to $\mathbb{R}_{+}^{d+1}$. Then there exists a positive constant $C$, depending only on $d$ and $\alpha$, such that

$$
|\nabla \Phi(x, y)| \leq C\left(\|\phi\|_{\infty}+\|\nabla \phi\|_{\infty}+\|\nabla \phi\|_{\alpha}\right)
$$

for all $(x, y) \in \mathbb{R}_{+}^{d+1}$.

Proof. First observe that, for $i=1, \ldots, d, \partial \Phi / \partial x_{i}$ is the Poisson extension of $\partial \phi / \partial x_{i}$. This shows that

$$
\left\|\frac{\partial \Phi}{\partial x_{i}}\right\|_{\infty} \leq\left\|\frac{\partial \phi}{\partial x_{i}}\right\|_{\infty}
$$

if $i=1, \ldots, d$. Now we estimate $\partial \Phi / \partial y$. For the case $y \geq 1$, we use the representation

$$
\frac{\partial \Phi}{\partial y}(x, y)=\frac{1}{2} \int_{\mathbb{R}^{d}} P_{y}(z, y)[\phi(x+z)+\phi(x-z)-2 \phi(x)] d z,
$$

which follows from differentiation of the Poisson integral formula and the symmetry properties of the Poisson kernel. Then

$$
\left|\frac{\partial \Phi}{\partial y}(x, y)\right| \leq A_{d}\|\phi\|_{\infty} \int_{\mathbb{R}^{d}} \frac{d z}{\left(1+|z|^{2}\right)^{(d+1) / 2}} \leq B_{d}\|\phi\|_{\infty} .
$$

Now assume $y<1$. Since for any $i=1,2, \ldots, d$, the function $\partial \phi / \partial x_{i}$ is in $\Lambda_{\alpha}\left(\mathbb{R}^{d}\right)$, its Poisson extension $\partial \Phi / \partial x_{i}$ satisfies

$$
\sup \left\{y^{1-\alpha}\left\|\nabla\left(\frac{\partial \Phi}{\partial x_{i}}(x, y)\right)\right\|:(x, y) \in \mathbb{R}_{+}^{d+1}\right\}<C(d)\left(\|\nabla \phi\|_{\alpha}+\|\nabla \phi\|_{\infty}\right)
$$

(see [17], p. 142). Hence,

$$
\sup \left\{y^{1-\alpha}\left\|\frac{\partial^{2} \Phi}{\partial^{2} y}(x, y)\right\|:(x, y) \in \mathbb{R}_{+}^{d+1}\right\}<C_{1}(d)\left(\|\nabla \phi\|_{\alpha}+\|\nabla \phi\|_{\infty}\right) .
$$

Integrating we deduce that if $0<y<1$, then $\partial \Phi / \partial y$ is uniformly bounded by $C_{2}(\alpha, d)\left(\|\nabla \phi\|_{\alpha}+\|\nabla \phi\|_{\infty}\right)$.

Actually the proof gives the stronger result

$$
\sup \left\{y^{1-\alpha}\|H \Phi(x, y)\|:(x, y) \in \mathbb{R}_{+}^{d+1}\right\}<\infty .
$$

Let $\phi: \mathbb{R}^{d} \rightarrow \mathbb{R}$ be a bounded function, let $\Phi$ be the Poisson extension of $\phi$ to $\mathbb{R}_{+}^{d+1}$, and let $H \Phi(x, y)$ the $(d+1) \times(d+1)$ Hessian matrix of $\Phi$ at $(x, y)$. Let $\|H \Phi(x, y)\|$ be as in (4.1). The next proposition provides sufficient conditions on $\phi$ for the boundedness of $\|H \Phi\|$. Let $\|H \phi\|_{\alpha}$ be the sum of the Hölder norms of all second order partial derivatives of the function $\phi$. 
Proposition 6.2. Let $\phi$ be a function in the class $C^{2, \alpha}\left(\mathbb{R}^{d}\right)$. Then there is a positive constant $C$, depending only on $d$ and $\alpha$, such that

$$
\|H \Phi(x, y)\| \leq C\left(\|\nabla \phi\|_{\infty}+\|H \phi\|+\|H \phi\|_{\alpha}\right)
$$

for any $(x, y) \in \mathbb{R}_{+}^{d+1}$.

Proof. It is easy to check that $\partial \Phi / \partial x_{i}$ (resp. $\left.\partial^{2} \Phi /\left(\partial x_{i} \partial x_{j}\right)\right)$ is the Poisson integral of $\partial \phi / \partial x_{i}\left(\operatorname{resp} . \partial^{2} \phi /\left(\partial x_{i} \partial x_{j}\right)\right), i=1, \ldots, d$. It follows then that

$$
\left\|\frac{\partial^{2} \Phi}{\partial x_{i} \partial x_{j}}\right\|_{\infty}=\left\|\frac{\partial^{2} \phi}{\partial x_{i} x_{j}}\right\|_{\infty}<\infty, \quad i, j=1, \ldots, d .
$$

For $\partial^{2} \Phi /\left(\partial y \partial x_{i}\right)$, apply Proposition 6.1 to $\partial \phi / \partial x_{i}$ instead of $\phi$. Finally, the estimate for $\partial^{2} \Phi / \partial y^{2}$ follows from harmonicity.

We will need the following result which says that the Poisson extension of a periodic function and its derivatives decay exponentially at infinity. It is valid under more general assumptions but this version will be sufficient for applications.

Proposition 6.3. Let $\phi: \mathbb{R}^{d} \rightarrow \mathbb{R}$ be a function in $C^{2, \alpha}\left(\mathbb{R}^{d}\right)$. Assume that $\phi$ is 1 periodic in each variable, that is, $\phi\left(x+e_{i}\right)=\phi(x)$ for all $x \in \mathbb{R}^{d}$ and any vector $e_{i}$ of the canonical basis in $\mathbb{R}^{d}, i=1, \ldots, d$. Let $\Phi(x, y)$ be the Poisson extension of $\phi$ to $\mathbb{R}_{+}^{d+1}$. Then there are positive constants $C=C(d), C_{1}=C_{1}(d, \alpha), C_{2}=C_{2}(d)$, such that, for any $(x, y) \in \mathbb{R}_{+}^{d+1}$,

$$
\begin{aligned}
& \left|\Phi(x, y)-C \int_{Q_{0}} \phi(z) d z\right| \leq C_{1}\|\phi\|_{\infty} e^{-C_{2} y}, \\
& |\nabla \Phi(x, y)| \leq C_{1}\left(\|\phi\|_{\infty}+\|\nabla \phi\|_{\infty}+\|\nabla \phi\|_{\alpha}\right) e^{-C_{2} y}, \\
& \|H \Phi(x, y)\| \leq C_{1}\left(\|\nabla \phi\|_{\infty}+\|H \phi\|_{\infty}+\|H \phi\|_{\alpha}\right) e^{-C_{2} y},
\end{aligned}
$$

where $Q_{0}=[0,1]^{d}$ is the unit cube in $\mathbb{R}^{d}$.

Proof. We use the Poisson summation formula (see [8])

$$
\sum_{k \in \mathbb{Z}^{d}} \frac{y}{\left(|x+k|^{2}+y^{2}\right)^{(d+1) / 2}}=\sum_{k \in \mathbb{Z}^{d}} e^{-2 \pi y|k|} e^{-2 \pi i<k, x>},
$$

that holds for any $(x, y) \in \mathbb{R}_{+}^{d+1}$. Then, by periodicity, there is a $C=C(d)$ such that

$$
\Phi(x, y)=C \int_{Q_{0}} \sum_{k \in \mathbb{Z}^{d}} \frac{y}{\left(|x-z+k|^{2}+y^{2}\right)^{(d+1) / 2}} \phi(z) d z
$$

so, by the Poisson formula,

$$
\Phi(x, y)=C\left(\int_{Q_{0}} \phi(z) d z+\sum_{k \in \mathbb{Z}^{d} \backslash\{0\}} e^{-2 \pi y|k|} \int_{Q_{0}} e^{-2 \pi i<k, x-z>} \phi(z) d z\right)
$$


Suppose first that $y \geq 1$. Then

$$
\begin{aligned}
1+\sum_{k \in \mathbb{Z}^{d} \backslash\{0\}} e^{-2 \pi y|k|} & \leq \sum_{k \in \mathbb{Z}^{d}} e^{-2 \pi y\left(\left|k_{1}\right|+\cdots+\left|k_{d}\right|\right) d^{-1 / 2}} \\
& =\left(\sum_{n=-\infty}^{\infty} e^{-2 \pi y|n| d^{-1 / 2}}\right)^{d}=\left(\frac{1+e^{-2 \pi y d^{-1 / 2}}}{1-e^{-2 \pi y d^{-1 / 2}}}\right)^{d},
\end{aligned}
$$

and, therefore,

$$
\left|\Phi(x, y)-C \int_{Q_{0}} \phi(z) d z\right| \leq\|\phi\|_{\infty}\left[\left(\frac{1+e^{-2 \pi y d^{-1 / 2}}}{1-e^{-2 \pi y d^{-1 / 2}}}\right)^{d}-1\right] \leq C_{1} e^{-C_{2} y}\|\phi\|_{\infty}
$$

as soon as $y \geq 1$, where $C_{1}$, and $C_{2}$ depend only on $d$. If $0<y<1$, the maximum principle gives $|\Phi(x, y)| \leq\|\phi\|_{\infty}$. This proves (6.1). To prove (6.2), we differentiate in (6.4) to obtain

$$
|\nabla \Phi(x, y)| \leq\|\phi\|_{\infty} \sum_{k \in \mathbb{Z}^{d} \backslash\{0\}}|k| e^{-2 \pi y|k|} .
$$

Suppose that $y \geq 1$. Then, since $t e^{-2 \pi y t} \leq e^{-\pi y t}$ if $t \geq 1$ we have

$$
\sum_{k \in \mathbb{Z}^{d} \backslash\{0\}}|k| e^{-2 \pi y|k|} \leq \sum_{k \in \mathbb{Z}^{d}} e^{-\pi y|k|}-1 \leq C_{1} e^{-C_{2} y},
$$

as above. If $0<y<1$, use Proposition 6.1. Essentially the same argument, together with Proposition 6.2, proves (6.3).

Lemma 6.5 below relates the differentiability properties of a function $f$ in $\mathbb{R}^{d}$ to the boundary behavior of the Hessian of its harmonic extension $\mathrm{F}$ to the upper-half space $\mathbb{R}_{+}^{d+1}$. Suppose that $F: \mathbb{R}_{+}^{d+1} \rightarrow \mathbb{R}$ is smooth, $t>0$ and $e \in \mathbb{R}^{d+1} \backslash \mathbb{R}^{d} \times\{0\}$ is unitary. Then it is easy to check that

$$
\begin{aligned}
\frac{\partial}{\partial t}(F(x+t e)) & =\nabla F(x+t e) \cdot e, \\
\frac{\partial^{2}}{\partial t^{2}}(F(x+t e)) & =\left(e^{T}(H F) e\right)(x+t e),
\end{aligned}
$$

where $H F$ is the Hessian matrix of $F$ and we interpret $e^{T}(H F) e$ in matrix form, $e^{T}$ being the vector $e$ written in row form. We need the following technical proposition, which is a generalization of formula (2.1).

Proposition 6.4. Let $f \in \Lambda_{*}\left(\mathbb{R}^{d}\right)$ and let $F$ be its Poisson extension to $\mathbb{R}_{+}^{d+1}$. Let $e \in \mathbb{R}^{d+1} \backslash \mathbb{R}^{d} \times\{0\}$ be a unitary vector. Then, for any $x \in \mathbb{R}^{d}$ and any $y>0$, there holds the identity

$$
f(x)=\int_{0}^{y} t\left(e^{T}(H F) e\right)(x+t e) d t-y \nabla F(x+y e) \cdot e+F(x+y e) .
$$


Proof. Since $\left(e^{T}(H F) e\right)(x+t e)=\frac{\partial^{2}}{\partial t^{2}} F(x+t e)$, integration by parts gives

$$
\begin{aligned}
\int_{0}^{y} t\left(e^{T}(H F) e\right)(x+t e) d t & =\left.t \frac{\partial}{\partial t} F(x+t e)\right|_{0} ^{y}-\int_{0}^{y} \frac{\partial}{\partial t} F(x+t e) d t \\
& =y \nabla F(x+y e) \cdot e-(F(x+y e)-f(x)),
\end{aligned}
$$

which implies (6.7). Observe that, from Proposition 2.1 and the hypothesis on $F$, $t \frac{\partial F}{\partial t}(x+t e) \rightarrow 0$ and $F(x+t e) \rightarrow f(x)$ as $t \rightarrow 0$.

Lemma 6.5. Let $f \in \Lambda_{*}\left(\mathbb{R}^{d}\right)$ and let $F$ be the Poisson extension of $f$ to $\mathbb{R}_{+}^{d+1}$. Suppose that there are a unitary vector $e \in \mathbb{R}^{d+1}$ and an open cube $Q \subset \mathbb{R}^{d}$ for which

$$
\sup _{(x, y) \in Q \times(0,1]}|(H F) e(x, y)|<\infty .
$$

Then:

1) If $e=(\hat{e}, 0) \in \mathbb{R}^{d} \times\{0\}$, both limits

$$
\lim _{t \rightarrow 0} \frac{f(x+t \hat{e})-f(x)}{t} \text { and } \lim _{t \rightarrow 0} \nabla F(x, t) \cdot e
$$

exist for any $x \in Q$.

2) If $e \in \mathbb{R}^{d+1} \backslash \mathbb{R}^{d} \times\{0\}$, then, for any $i=1, \ldots, d$,

$$
\frac{\partial f}{\partial x_{i}}(x) \text { and } \lim _{t \rightarrow 0} \frac{\partial F}{\partial x_{i}}(x+t e)
$$

exist for any $x \in Q$.

Proof. By (6.8), for any $x \in Q$, one has

$$
\sup _{0<y<1}\left|\frac{\partial}{\partial y} \nabla F(x, y) \cdot e\right|<\infty,
$$

which implies the existence of $\lim _{y \rightarrow 0} \nabla F(x, y) \cdot e$.

Assume first that $e=(\hat{e}, 0) \in \mathbb{R}^{d} \times\{0\}$. Apply Proposition 6.4 to the unit normal vector in the $y$-direction. Then

$$
f(x+t \hat{e})-f(x)=I_{1}+I_{2}+I_{3},
$$

where

$$
\begin{aligned}
I_{1} & =\int_{0}^{t} y\left[\frac{\partial^{2} F}{\partial y^{2}}(x+t \hat{e}, y)-\frac{\partial^{2} F}{\partial y^{2}}(x, y)\right] d y, \\
I_{2} & =t\left[\frac{\partial F}{\partial y}(x+t \hat{e}, t)-\frac{\partial F}{\partial y}(x, t)\right], \\
I_{3} & =F(x+t \hat{e}, t)-F(x, t) .
\end{aligned}
$$


From (6.8) and the Cauchy estimates for harmonic functions it follows that

$$
\sup _{Q \times(0,1]} y\left|\frac{\partial^{2}}{\partial y^{2}} \nabla F(x, y) \cdot e\right|<\infty,
$$

which implies that $\left|I_{1}\right| \leq C t^{2}$. By (6.8), $\left|I_{2}\right| \leq C t^{2}$, so both $I_{1}$ and $I_{2}$ are $o(t)$ as $t \rightarrow 0$. Finally, observe that

$$
\frac{I_{3}}{t}=\frac{1}{t} \int_{0}^{t} \nabla F(x+s \hat{e}, t) \cdot e d s
$$

and that, again from (6.8),

$$
|\nabla F(x+s \hat{e}, t)-\nabla F(x, t)| \leq C s .
$$

Therefore we deduce that $I_{3} / t$ has a limit as $t \rightarrow 0$ and part 1) follows.

Now assume $e \in \mathbb{R}^{d+1} \backslash \mathbb{R}^{d}$. Fix $i=1, \ldots, d$. From (6.8) we get

$$
\left|\frac{\partial F}{\partial x_{i}}(x+t e)-\frac{\partial F}{\partial x_{i}}(x+s e)\right| \leq C|t-s|,
$$

so $\lim _{t \rightarrow 0} \frac{\partial F}{\partial x_{i}}(x+t e)$ exists. Now for $t>0$, choose $M=M(t)>0$ so that $M(t) \rightarrow \infty$ and $t M(t) \rightarrow 0$ as $t \rightarrow 0$. From Proposition 6.4 we get

$$
\begin{aligned}
f\left(x+t e_{i}\right)-f(x)= & \int_{0}^{M t} s\left[\left(e^{T}(H F) e\right)\left(x+t e_{i}+s e\right)-\left(e^{T}(H F) e\right)(x+s e)\right] d s \\
& -t M\left[\nabla F\left(x+t e_{i}+t M e\right) \cdot e-\nabla F(x+t M e) \cdot e\right] \\
& +F\left(x+t e_{i}+t M e\right)-F(x+t M e)=I_{1}-I_{2}+I_{3} .
\end{aligned}
$$

As before, Cauchy's estimates give that

$$
\left|\left(e^{T}(H F) e\right)\left(x+t e_{i}+s e\right)-\left(e^{T}(H F) e\right)(x+s e)\right| \leq C t / s
$$

so $\left|I_{1}\right| \leq C M t^{2}$. In the same way, $\left|I_{2}\right| \leq C M t^{2}$. As for $I_{3}$,

$$
\frac{I_{3}}{t}=\frac{1}{t} \int_{0}^{t} \frac{\partial F}{\partial x_{i}}\left(x+s e_{i}+t M e\right) d s
$$

and, from Propositions 2.1 and 2.2,

$$
\left|\frac{\partial F}{\partial x_{i}}\left(x+s e_{i}+t M e\right)-\frac{\partial F}{\partial x_{i}}(x+t M e)\right| \leq C\|f\|_{*} \frac{s}{t M} \leq \frac{C\|f\|_{*}}{M} .
$$

Therefore, for any $0<s<t$,

$$
\frac{I_{3}}{t}=\frac{\partial F}{\partial x_{i}}(x+t M e)+O\left(\frac{1}{M}\right) .
$$

The lemma follows from the choice of $M$ and the existence of $\lim _{t \rightarrow 0} \partial F / \partial x_{i}(x+t e)$. 


\section{Functions of Weierstrass type}

Now we turn to functions of Weierstrass type. Fix $b>1$ and let $\phi: \mathbb{R}^{d} \rightarrow \mathbb{R}$ be as in the statement of Theorem 3. Let

$$
f(x)=f_{b, \phi}(x)=\sum_{n=0}^{\infty} b^{-n} \phi\left(b^{n} x\right)
$$

be the Weierstrass function associated to $b$ and $\phi$. Denote by $\Phi$ (resp. $F$ ) the Poisson extension of $\phi$ (resp. $f$ ) to $\mathbb{R}_{+}^{d+1}$. Then

$$
F(x, y)=\sum_{n=0}^{\infty} b^{-n} \Phi\left(b^{n} x, b^{n} y\right)
$$

which leads to the functional equation (7.3) here that will be used later:

$$
F(b x, b y)=b F(x, y)-b \Phi(x, y),
$$

and by differentiation, to the equations

$$
\begin{aligned}
\nabla F(b x, b y) & =\nabla F(x, y)-\nabla \Phi(x, y), \\
b H F(b x, b y) & =H F(x, y)-H \Phi(x, y) .
\end{aligned}
$$

Proposition 7.1. Let $f(r e s p . F)$ be as in (7.1) (resp. (7.1)). Then $f \in \Lambda_{*}\left(\mathbb{R}^{d}\right)$ and

$$
\|f\|_{*} \leq C_{1}\left(\|\phi\|_{\infty}+\|H \phi\|_{\infty}\right) \text {. }
$$

Furthermore,

$$
\sup \left\{y\|H F(x, y)\|:(x, y) \in \mathbb{R}_{+}^{d+1}\right\} \leq C_{2}\left(\|\phi\|_{\infty}+\|H \phi\|_{\infty}\right),
$$

where $C_{1}$ and $C_{2}$ are positive constants depending only on $d$ and $b$.

Proof. The fact that $f \in \Lambda_{*}\left(\mathbb{R}^{d}\right)$ uses a trick standard in the theory of lacunary series. If $h \in \mathbb{R}^{d}$, denote $\Delta_{h}^{2} f(x)=f(x+h)+f(x-h)-2 f(x)$. If $|h| \geq 1$, we have

$$
\left\|\Delta_{h}^{2} f\right\|_{\infty} \leq 4\|f\|_{\infty} \leq C(b)|h|\|\phi\|_{\infty},
$$

so we can assume that $|h|<1$. Choose $N \geq 0$ so that $b^{-(N+1)}<|h| \leq b^{-N}$. A simple computation shows that if $a, h \in \mathbb{R}^{d}$ then

$$
\left|\Delta_{h}^{2} \phi(a)\right| \leq C(d)\|H \phi\|_{\infty}|h|^{2} .
$$

Now split $\Delta_{h}^{2} f(x)=A+B$, where

$$
A=\sum_{n=0}^{N} b^{-n} \Delta_{b^{n} h}^{2} \phi\left(b^{n} x\right) \text { and } B=\sum_{n=N+1}^{\infty} b^{-n} \Delta_{b^{n} h}^{2} \phi\left(b^{n} x\right) .
$$

By (7.6) and the choice of $N$ we get that $|A| \leq C(d, b)|h|\|H \phi\|_{\infty}$. On the other hand, $|B| \leq 4 b^{-N}(b-1)^{-1}\|\phi\|_{\infty} \leq C(b)|h|\|\phi\|_{\infty}$. Hence $\|f\|_{*} \leq C_{1}\left(\|\phi\|_{\infty}+\right.$ $\left.\|H \phi\|_{\infty}\right)$. The last assertion follows from Proposition 2.2. 
We recall now condition $\mathcal{H}$ on $\phi$, which, in the case $d=1$, was used by Heurteaux (see Theorem 3.1 in [10]). We say that $\phi$ satisfies condition $\mathcal{H}$ if, for any unitary vector $e \in \mathbb{R}^{d}$, either $D_{e} \phi(0) \neq 0$ or the one-variable function $t \rightarrow \phi(t e)$, is nonconstant and has a global extremum at $t=0$.

Proposition 7.2. Let $b>1$ and let $\phi$ be as in Proposition 6.3. Let $f=f_{b, \phi}$ be the Weierstrass-type function associated to $b$ and $\phi$ defined by (7.1) and let $F$ be the Poisson extension of $f$ to $\mathbb{R}_{+}^{d+1}$. Assume, additionally, that $\phi$ satisfies condition $\mathcal{H}$. Then for any $M>0$ there exists $\eta, 0<\eta<1$, such that

$$
\inf _{|e|=1} \sup _{(x, y) \in Q_{0} \times[\eta, 1]}|(H F) e(x, y)|>M,
$$

where $Q_{0}=[-1 / 2,1 / 2]^{d}$ and the infimum is taken over all unitary $e \in \mathbb{R}^{d+1}$.

Proof. Suppose that the conclusion does not hold. Then there are $M>0$ and a sequence $\left\{e_{n}\right\}$ of unitary vectors in $\mathbb{R}^{d+1}$ such that

$$
\sup _{(x, y) \in Q_{0} \times[1 / n, 1]}\left|(H F) e_{n}(x, y)\right| \leq M .
$$

Taking a subsequence if necessary we can assume that $e_{n} \rightarrow e$, where $e \in \mathbb{R}^{d+1}$ is also unitary and satisfies

$$
\sup _{(x, y) \in Q_{0} \times(0,1]}|(H F) e(x, y)| \leq M .
$$

We will apply Lemma 6.5 only in the case $x=0$. If $e=(\hat{e}, 0) \in \mathbb{R}^{d} \times\{0\}$ then, by Lemma 6.5 , the functional equation (7.4), and the fact that $\nabla \Phi$ is continuous up to the boundary, we get

$$
\nabla \Phi(0,0) \cdot e=\nabla \phi(0) \cdot \hat{e}=0 .
$$

The rest of the argument follows [10]. By condition $\mathcal{H}$, the function $\phi(t \hat{e})$ is nonconstant and has a global extremum, say a global maximum, at $t=0$. In particular, $f(t \hat{e})$ has also a global maximum at $t=0$ and, by Lemma 6.5 ,

$$
\lim _{t \rightarrow 0} \frac{f(t e)-f(0)}{t}=0 .
$$

Fix $t \in \mathbb{R}$. Now for each positive integer $n$,

$$
f\left(b^{-n} t \hat{e}\right)-f(0)=\sum_{k=0}^{\infty} b^{-k}\left(\phi\left(b^{k-n} t \hat{e}\right)-\phi(0)\right) \leq b^{-n}(\phi(t \hat{e})-\phi(0)) .
$$

Therefore,

$$
0=\lim _{n \rightarrow \infty} \frac{f\left(b^{-n} t \hat{e}\right)-f(0)}{b^{-n}} \leq \phi(t \hat{e})-\phi(0),
$$

which contradicts the fact that $\phi(t e)$ is nonconstant and has a global maximum at $t=0$. 
If $e \in \mathbb{R}^{d+1} \backslash \mathbb{R}^{d}$ then we deduce, again from Lemma 6.5, that

$$
\lim _{t \rightarrow 0} \frac{\partial \Phi}{\partial x_{i}}(t e)=\frac{\partial \phi}{\partial x_{i}}(0)=0
$$

for each $i=1,2, \ldots, d$, so $\nabla \phi(0)=0$. The same argument above, applied to any of the coordinate directions, provides a contradiction as well.

We are now ready to prove that, provided $\phi$ satisfies condition $\mathcal{H}$, then the gradient of the Poisson extension of $f_{b, \phi}$ defined by (7.1) satisfies a $(1 / N)$-weak QR condition for some integer $N \geq 2$. We need to recall the concept of an almost periodic function and some of the basic properties of such functions.

Given $g: \mathbb{R}^{d} \rightarrow \mathbb{R}$ and $\epsilon>0$, we say that $\tau \in \mathbb{R}^{d}$ is an almost period of $g$ relative to $\epsilon$ if

$$
\sup \left\{|g(x+\tau)-g(x)|: x \in \mathbb{R}^{d}\right\} \leq \epsilon .
$$

A continuous function $g: \mathbb{R}^{d} \rightarrow \mathbb{R}$ is said to be almost periodic (a.p.) if for any $\epsilon>0$ there exists $l>0$ such that any cube $Q \subset \mathbb{R}^{d}$ of sidelength $l$ contains an almost period of $g$ relative to $\epsilon$. A mapping $g: \mathbb{R}^{d} \rightarrow \mathbb{R}^{d}$ is almost periodic if so is each of its components. As in the classical case $d=1$, almost periodic functions in $\mathbb{R}^{d}$ turn out to be those that can be uniformly approximated by trigonometric polynomials. Any continous function which is periodic in each variable is almost periodic in $\mathbb{R}^{d}$. It can also be shown that finite sums and uniform limits of almost periodic functions are almost periodic too. We refer to the classical monograph by Besicovitch ([3]) for these and other results.

Now let $\phi$ and $f_{b, \phi}$ and their respective Poisson extensions $\Phi$ and $F$ be as in the beginning of the section. Differentiating (7.2) twice we get

$$
H F(x, y)=\sum_{n=0}^{\infty} b^{n} H \Phi\left(b^{n} x, b^{n} y\right),
$$

where the series converges uniformly on any strip $0<a \leq y \leq b$ thanks to the exponential decay provided by Proposition 6.3. In the case of interest, $0<a=$ $\eta(\phi, b, d)<1=b$. Now, for $\eta \leq y \leq 1$ and unitary $e \in \mathbb{R}^{d+1}$, consider the mapping

$$
x \rightarrow(H F) e(x, y), \quad\left(x \in \mathbb{R}^{d}\right) .
$$

Since, for each $n \geq 0, H \Phi\left(b^{n} x, b^{n} y\right) e$ is $b^{-n}$-periodic in $x$, it follows from uniform convergence that $(H F) e(x, y)$ is a. p. in $x$. But actually, a bit more is true. From the inequality

$$
|(H F) e(x+\tau, y)-(H F) e(x, y)| \leq\|H F(x+\tau, y)-H F(x, y)\|
$$

and the basic properties of almost periodic functions it can be shown that the almost periodicity of $x \rightarrow(H F) e(x, y)$ is uniform in $e$ and $y \in[\eta, 1]$ in the sense that, given $\epsilon>0$, the $l$ in the definition of almost periodicity will depend only on $\epsilon, \phi, b$, and $d$ but not on $e$. This fact will be useful in the proof of the following lemma.

For $0<\delta<1$ and any cube $Q \subset \mathbb{R}^{d}$ as in Section 4 , we denote by $C_{\delta}(Q)=$ $Q \times[\delta l(Q), l(Q)]$ the $\delta$-Carleson box associated to $Q$. 
Lemma 7.3. Let $\phi: \mathbb{R}^{d} \rightarrow \mathbb{R}$ be as in Proposition 6.3. Assume in addition that $\phi$ satisfies condition $\mathcal{H}$. For $b>1$, let $f$ be the Weierstrass function associated to $b$ and $\phi$ as in (7.1) and let $F$ be the Poisson extension of $f$ given by (7.2). Then there are positive constants $\delta=\delta(\phi, b)<1$ and $c=c(\phi, b)$ such that, for any cube $Q \subset \mathbb{R}^{d}$ of sidelength $l(Q) \leq 1$,

$$
\inf _{|e|=1} \sup _{(x, y) \in C_{\delta}(Q)}|(H F) e(x, y)| \geq \frac{c}{l(Q)},
$$

where the infimum is taken over all unitary vectors $e \in \mathbb{R}^{d+1}$.

Proof. From the functional equation (7.5) for the Hessian we get

$$
(H F) e\left(b^{-1} x, b^{-1} y\right)=b(H F) e(x, y)+(H \Phi) e\left(b^{-1} x, b^{-1} y\right) .
$$

Iterating (7.7) we obtain

$$
(H F) e\left(b^{-k} x, b^{-k} y\right)=b^{k}(H F) e(x, y)+\sum_{n=0}^{k-1} b^{n}(H \Phi) e\left(b^{n-k} x, b^{n-k} y\right)
$$

for any nonnegative integer $k=1,2, \ldots$ Therefore,

$$
\left|(H F) e\left(b^{-k} x, b^{-k} y\right)\right| \geq b^{k}\left(|(H F) e(x, y)|-\frac{\|H \Phi\|}{b-1}\right) .
$$

By Proposition 7.2 there exists $0<\eta<1$ so that for any unitary vector $e \in \mathbb{R}^{d+1}$ there exist $x_{0} \in Q_{0}=[-1 / 2,1 / 2]^{d}$ and $\eta<y_{0}<1$ such that

$$
\left|(H F) e\left(x_{0}, y_{0}\right)\right|>\frac{3\|H \Phi\|_{\infty}}{b-1} .
$$

Now, by the previous remarks, the function $x \rightarrow(H F) e\left(x, y_{0}\right)$ is a.p., uniformly in $e$ and $y_{0}$ so we can choose $l=l(\phi, b, d) \geq 1$ such that for any cube $Q^{\prime} \subset \mathbb{R}^{d}$ with $l\left(Q^{\prime}\right) \geq l$ there is an almost period $\tau \in Q^{\prime}$ relative to $\epsilon=\|H \Phi\|_{\infty} /(b-1)$ that is uniform respect to $e$. In other words,

$$
\left|(H F) e\left(x+\tau, y_{0}\right)-(H F) e\left(x, y_{0}\right)\right| \leq \frac{\|H \Phi\|_{\infty}}{b-1}
$$

for any $x \in \mathbb{R}^{d}$ and any unitary $e \in \mathbb{R}^{d+1}$. Fix a nonnegative integer $k$ such that $b^{-k} l<l(Q) \leq b^{-k+1} l$. Let $Q^{\prime}=b^{k} Q-x_{0}$ where, for $a>0, a Q=\{a x: x \in Q\}$. Then $l\left(Q^{\prime}\right) \geq l$ so there is $\tau \in Q^{\prime}$ satisfying (7.10). Observe that $b^{-k}\left(x_{0}+\tau\right) \in Q$. From (7.8), (7.9) and (7.10) we get

$$
b^{-k} y_{0}\left|(H F) e\left(b^{-k}\left(x_{0}+\tau, y_{0}\right)\right)\right| \geq y_{0}\left[\left|(H F) e\left(x_{0}+\tau, y_{0}\right)\right|-\frac{\|H \Phi\|_{\infty}}{b-1}\right] \geq \eta \frac{\|H \Phi\|_{\infty}}{b-1} .
$$

On the other hand,

$$
\frac{\eta}{b l} l(Q)<b^{-k} y_{0}<l(Q),
$$

so the conclusion follows upon taking $\delta=\eta /(b l)$ and $c=\eta\|H \Phi\|_{\infty} /(b-1)$. 
Corollary 7.4. Let $b, \phi, f$, and $F$ be as in Lemma 7.3. Then there are $N=$ $N(\phi, b, d) \in \mathbb{N}, N \geq 2$, and $\gamma=\gamma(\phi, b, d) \geq 1$ such that $\nabla F$ verifies a $1 / N$-weak $Q R$ condition with constant $\gamma$. That is, for any cube $Q \subset \mathbb{R}^{d}$,

$$
\int_{C_{1 / N}(Q)} \max _{|e|=1}|H F(x, y)|^{2} d x d y \leq \gamma^{2} \min _{|e|=1} \int_{C_{1 / N}(Q)}|(H F) e(x, y)|^{2} d x d y .
$$

Proof. Given a cube $Q$ in $\mathbb{R}^{d}$, let $\widetilde{Q}$ be the cube with the same center and half its sidelength. By Lemma 7.3, there are $0<\delta=\delta(\lambda, \phi)<1$ and $c=c(\phi, b)>0$ such that for any unitary vector $e \in \mathbb{R}^{d+1}$ there exists a point $(x, y) \in C_{\delta}(\widetilde{Q})$ such that

$$
|(H F) e(x, y)| \geq \frac{2 c}{l(Q)} .
$$

Let $B \subset \mathbb{R}_{+}^{d+1}$ be the ball centered at $(x, y)$ of radius $\delta l(Q) / 4$. Then, from subharmonicity,

$$
|(H F) e(x, y)|^{2} \leq \frac{C_{1}}{l(Q)^{d+1}} \int_{B}|(H F) e|^{2}
$$

for some $C_{1}=C_{1}(d)$. Choose $N \in \mathbb{N}$ such that $1 / N \leq \delta / 4 \leq 1 /(N-1)$. Then $B \subset C_{1 / N}(Q)$ and, combining the two previous inequalities, we get

$$
C_{2}(l(Q))^{d-1} \leq \min _{|e|=1} \int_{C_{1 / N}(Q)}|(H F) e|^{2}
$$

for some constant $C_{2}=C_{2}(b, d, \phi)$. On the other hand, from Proposition 7.1,

$$
\sup _{C_{1 / N}(Q)}\|H F\|^{2} \leq \frac{C_{3}}{(l(Q))^{2}}
$$

where $C_{3}=C_{3}(b, d, \phi)$. In particular,

$$
\int_{C_{1 / N}(Q)}\|H F\|^{2} \leq C_{4}(l(Q))^{d-1} .
$$

The conclusion follows from (7.11) and (7.12).

\section{Proof of Theorem 3}

Proof. The fact that $f_{b, \phi} \in \Lambda_{*}\left(\mathbb{R}^{d}\right)$ follows from Proposition 7.1. The fact that $f_{b, \phi}$ is nowhere differentiable follows from Theorem 3.1 in [10]. Pick $x_{0} \in \mathbb{R}^{d}$. If $x_{0} \neq 0$, define $e=x_{0} /\left|x_{0}\right|$ and observe that the one-variable function $t \rightarrow f(t e)$ satisfies the hypothesis of Theorem 3.1 in [10] so is nowhere differentiable. In particular $f$ has no directional derivative at $x_{0}$ along the radial direction $e$. If $x_{0}=0$ the same argument shows that $f$ has no directional derivative at 0 along any direction. This proves part 1). 
Let $0<\delta<1$ and $c>0$ be the constants appearing in Lemma 7.3. Fix $x_{0} \in \mathbb{R}^{d}$ and a unitary vector $e \in \mathbb{R}^{d}$. Let $Q_{k}$ be the cube centered at $x_{0}$ and having sidelength $\delta^{k}$. By Lemma 7.3, there is $(x, y) \in Q_{k} \times\left[\delta^{k+1}, \delta^{k}\right]$ such that $|H F \cdot e(x, y)| \geq c / y$. Let $B_{k}$ be the ball centered at $(x, y)$ of radius $\delta^{k} / 2$. By subharmonicity,

$$
\frac{C}{\delta^{2 k}} \leq \frac{1}{\delta^{(k+1)(d+1)}} \int_{B_{k}}\left|\nabla\left(D_{e} F\right)\right|^{2} d x d y,
$$

where $D_{e} F$ means the derivative of $F$ in the direction $e$ and $C=C(d, b, \phi)$. It is easy to check that

$$
B_{k} \subset Q_{k}^{\prime} \times\left[\frac{\delta^{k+1}}{2}, \delta^{k}-\frac{\delta^{k+1}}{2}\right] \subset \Gamma\left(x_{0}\right)
$$

where

$$
\Gamma\left(x_{0}\right)=\left\{(x, y) \in \mathbb{R}_{+}^{d+1}:\left|x-x_{0}\right| \leq(1+\sqrt{d} / \delta) y\right\} .
$$

On the other hand, from (8.1) and (8.2) we get

$$
\int_{B} y^{1-d}\left|\nabla\left(D_{e} F\right)(x, y)\right|^{2} d x d y \geq C>0
$$

for some $C>0$ independent of $k$. Since each cone $\Gamma\left(x_{0}\right)$ contains infinitely many disjoint balls $B_{k}$, we get from (8.3) that

$$
\int_{\Gamma\left(x_{0}\right)} y^{1-d}\left|\nabla\left(D_{e} F\right)(x, y)\right|^{2} d x d y=\infty,
$$

which implies that the area function of $D_{e} F$ for cones of some fixed aperture is infinite for all $x_{0} \in \mathbb{R}^{d}$. From the area version of the local Fatou theorem for harmonic functions (see Theorems 3 and 4 in Chapter VII of [17]), it follows that $D_{e} F$ is nontangentially unbounded at almost every point $x \in \mathbb{R}^{d}$. Part 2) of Theorem 3 now follows from Proposition 2.1 and Corollary 2.4.

To prove part 3) of Theorem 3 , let $b, \phi$, and $f_{b, \phi}$ be as in the statement of the theorem and let $F$ be the Poisson extension of $f_{b, \phi}$. Let $N \in \mathbb{N}$ and $\gamma \geq 1$ be as in Corollary 7.4. The result follows from Corollaries 3.8 and 4.5.

\section{Remarks and questions}

1) Quasi-regular mappings can be understood as a sort of higher-dimensional analogue of holomorphic mappings. On the other hand, in harmonic analysis, the higher-dimensional analogue of a holomorphic mapping is the gradients of harmonic functions, or equivalently, a system of conjugate harmonic functions (see [17], p. 65). Can one describe the harmonic functions in an upper half-space whose gradient is (weakly) quasi-regular? Quasi-regularity is typically an involved property to handle. If $u$ is harmonic in $\mathbb{R}^{d}$, for $d \geq 3$ and $u$ is independent of at least one direction, then it is clear that $\nabla u$ cannot be quasi-regular. To what extent is this the only obstacle for a harmonic gradient to be quasiregular? 
2) The weak quasiregularity condition used in section 4 raises some natural but subtle questions. Suppose that $\nabla F$ is a harmonic gradient. It is not clear (and probably false) that if $\nabla F$ satisfies a $\delta$ - weak QR condition then it also satisfies a $\delta^{\prime}$-weak $\mathrm{QR}$ condition for $\delta^{\prime} \simeq \delta$. The way to show that $\nabla F$ satisfies a weak QR condition (Corollary 7.4) relies on the functional equation (7.5) and on a certain lower uniform bound for the Hessian HF (Lemma 7.3) together with sub-harmonicity. This is a sort of bypass that avoids the problem of comparing directly the maximal and the minimal distortions of $\nabla F$. Because of this, we have been unable to adapt the method used to prove part 3) of Theorem 3 to cover the case of lacunary series in $\lambda_{*}$ of the form

$$
f(x)=\sum_{n=0}^{\infty} \varepsilon_{n} b^{-n} \phi\left(b^{n} x\right),
$$

where $\left\{\varepsilon_{n}\right\}$ is a sequence of real numbers tending to 0 .

3) The dichotomy given by Proposition 5.1 can fail dramatically if either harmonicity or quasiregularity is dropped from the hypothesis. Indeed, if $u$ is harmonic in $\mathbb{R}^{d}, d \geq 2$, and $u$ does not depend on one of the variables then $\nabla u$ is not quasiregular (even in the weak sense) and the conclusions of Proposition 5.1 obviously do not hold. On the other hand, there is a bounded quasiregular mapping $g: \mathbb{R}_{+}^{2} \rightarrow \mathbb{C}$ that fails to have vertical limit at almost all $x \in \mathbb{R}$, as the following construction shows. Let $h$ be an increasing, singular, quasisymmetric homeomorphism of $\mathbb{R}$ into $\mathbb{R}$ (see Theorem 3 in [4]). This implies the existence of $E \subset \mathbb{R}$ such that $m_{1}(\mathbb{R} \backslash E)=m_{1}(h(E))=0$. Extend $h$ to a quasiconformal map $H: \mathbb{R}_{+}^{2} \rightarrow \mathbb{R}_{+}^{2}$ (see Theorem 1 in [4]). Now take a bounded analytic function $f: \mathbb{R}_{+}^{2} \rightarrow \mathbb{C}$ such that for any $x \in h(E), f$ fails to have a limit along any curve ending at $x$ (see Lemma 1 in Chapter 2 of [6]). The statement follows by taking $g=f \circ H$ (see Theorem 5.5.1 in [4] for the quasiregularity of $g$ ).

4) Even if $d=1$, the authors wonder which part of the results in [10] can be saved if the base function $\phi$ is only assumed to be Lipschitz. On the other hand it is also natural to ask to what extent the periodicity or almost periodicity of $\phi$ is essential for the nowhere differentiability of the Weierstrass function.

\section{References}

[1] Anderson, J. M. And Pitt, L. D.: Probabilistic behaviour of functions in the Zygmund spaces $\Lambda^{*}$ and $\lambda^{*}$. Proc. London Math. Soc. 3 (1989), no. 3, 558-592.

[2] Astala, K., Iwaniec, T. And Martin, G.: Elliptic partial differential equations and quasiconformal mappings in the plane. Princeton Mathematical Series 48 , Princeton University Press, 2009.

[3] Besicovitch, A.S.: Almost periodic functions. Dover, 1955.

[4] Beurling, A. And Ahlfors, L. V.: The boundary correspondence under quasiconformal mappings. Acta Math. 96 (1956), 125-142.

[5] Carleson, L.: On the existence of boundary values for harmonic functions in several variables. Ark. Mat. 4 (1962), 393-399. 
[6] Collingwood, E. F. And Lohwater, A. J.: The theory of cluster sets. Cambridge University Press, 1966

[7] Donaire, J. J., Llorente, J. And Nicolau, A.: Differentiability of functions in the Zygmund class. Proc. London Math. Soc. (3) 108 (2014), no. 1, 133-158.

[8] Grafakos, L.: Classical Fourier analysis. Second edition. Graduate texts in Mathematics 249, Springer, 2008.

[9] Hardy, G.H.: Weierstrass' non-differentiable function. Trans. Amer. Math. Soc. 17 (1916), no. 3, 301-325.

[10] Heurteaux, Y.: Weierstrass functions in Zygmund's class. Proc. Amer. Math. Soc. 133 (2005), no. 9, 2711-2720.

[11] Kahane, J. P.: Sur l'irregularité locale du movement brownien. C. R. Acad. Sci. Paris Sér. A. 278 (1974), 331-333.

[12] Krantz, S. G.: Lipschitz spaces, smoothness of functions and approximation theory. Expo. Math. 1 (1983), no. 3, 193-260.

[13] Makarov, N. G.: On the radial behavior of Bloch functions. Dokl. Akad. Nauk SSSR (Russian) 309 (1989), no. 2, 275-278; translation in Soviet Math. Dokl. 40 (1990), no. 3, 505-508.

[14] Makarov, N. G.: Probability methods in conformal mappings. Algebra i Analiz (Russian) 1 (1989), no. 1, 3-59; translation in Leningrad Math. J. 1 (1990), no. 1, $1-56$.

[15] Pommerenke, Сн.: Boundary behaviour of conformal maps. Springer-Verlag, 1991.

[16] Rickman, S.: Quasiregular mappings. Springer-Verlag, 1993.

[17] Stein, E. M.: Singular integrals and differentiability properties of functions. Princeton University Press, 1970.

[18] Thim, J.: Continous nowhere differentiable functions. Master's thesis, Luleå University of Technology, 2003.

[19] Zygmund, A.: Smooth functions. Duke Math. J. 12 (1945), 45-76.

[20] Zygmund, A.: Trigonometric series. Vols. I, II. Cambridge University Press, 2002.

Received September 25, 2012; revised July 25, 2013.

Juan Jesús Donaire: Departament de Matemàtiques, Universitat Autònoma de Barcelona, Bellaterra 08193, Barcelona, Spain.

E-mail: donaire@mat.uab.cat

José G. Llorente: Departament de Matemàtiques, Universitat Autònoma de Barcelona, Bellaterra 08193, Barcelona, Spain.

E-mail: jgllorente@mat.uab.cat

Artur Nicolau: Departament de Matemàtiques, Universitat Autònoma de Barcelona, Bellaterra 08193, Barcelona, Spain.

E-mail: artur@mat.uab.cat

Partially supported by grants MTM2008-05561, 2009SGR1303, MTM2011-2406 and 2009SGR420. 